$$
\begin{aligned}
& \text { ATENCIÓN } \\
& \text { AL FINAL DE } \\
& \text { LA VIDA }
\end{aligned}
$$

MODELO ORGANIZATIVO DE EXTREMADURA 
Usted importa porque es usted y, seguirá importando hasta el

último momento de su vida.

Nosotros, no sólo le ayudaremos a

morir en paz, sino a vivir hasta que muera

Cicely Saunders 


\section{ATENCIÓN AL FINAL DE LA VIDA}


Titulo
Atenci

Autores
Programa

Edita
Osservatorio Regional de Cuidados Paliativos de Extremadura (ORCPEX).
(Junta de Extremadura, Servicio Extremeño de Salud - FundeSalud).

Depósito Legal CC-171-2011

Ejemplares 5.000
Edición Diciembre 2010

Proyecto Gráfico

jesusburgosberzosa@gmail.com

Imprime
Artes Gráficas Batanero SL.

Artes Graficas Batanero 5 .
www.agbatanero. com

Agradecemos I a colaboración de todas las personas que han participado en I a redacción y revi-
sión del documento (Grupo de Registro, observatorio Regional de Cuidados Paliativos, plan de Voluntariadol) y, especialmente, a todos los profesionales implicados en la atención al final de 


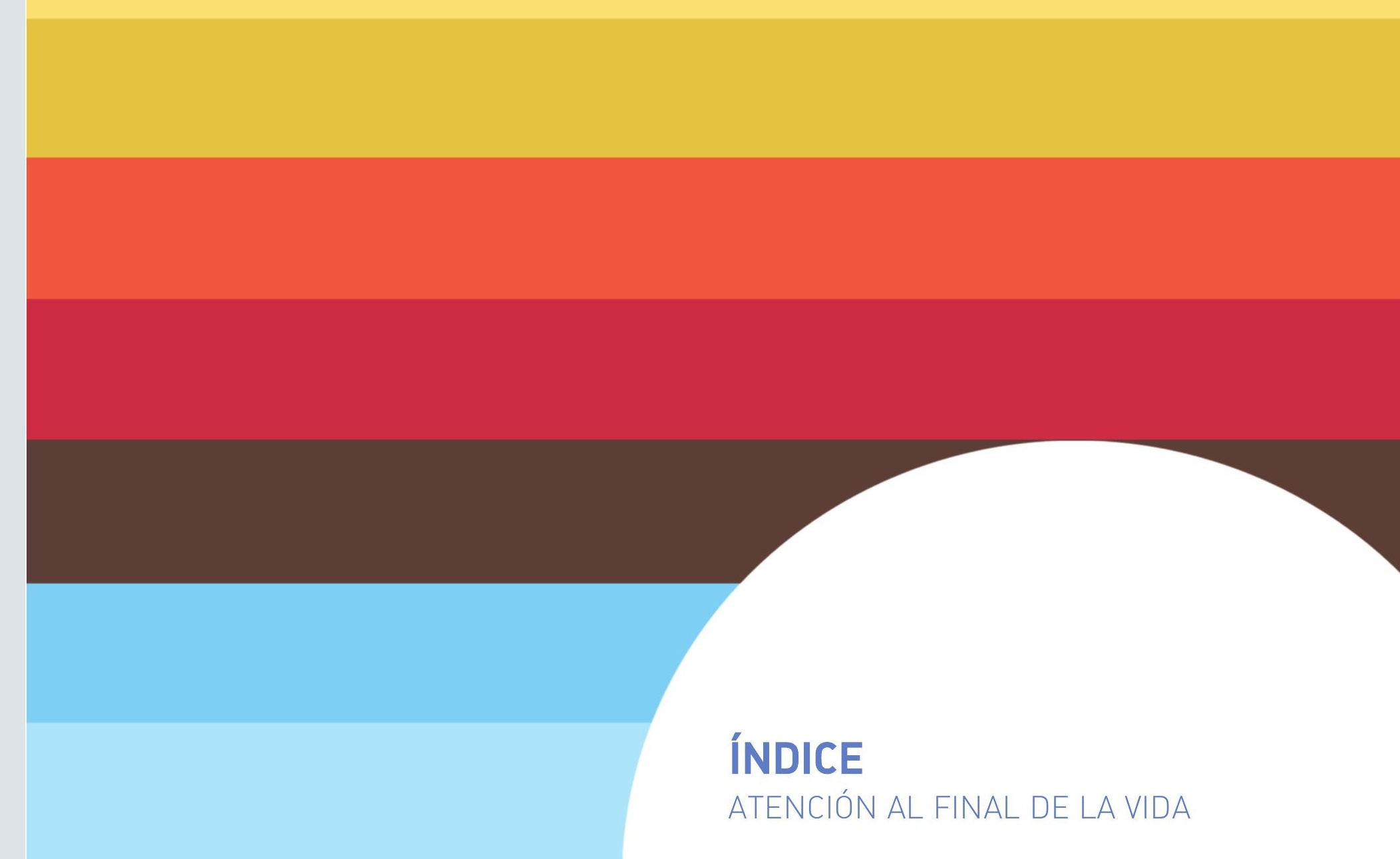


Introducción
Enfermedad

Patrones evolutivos de la enfermedad avanzada y termina Cuidados Paliativos

MODELO ORGANIZATIVO
DE EXTREMADURA

Modelo organizativo

Caracteristicas

Objetivos
Evaluación

RECURSOS GENERALES

Recursos generales
Medidas

RECURSOS ESPECÍFICOS

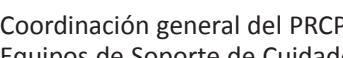

(ESCP)

Trabajo Transversales

Formación (FORPALEX)

Registro y Documentación Clínica (REGPALEX)

Calidad (CALPA

Investigación (IPALEX)

Observatorio Regional de Cuidados Paliativos (ORCPEx)
Plan de Voluntariado en Cuidados Paliativos 


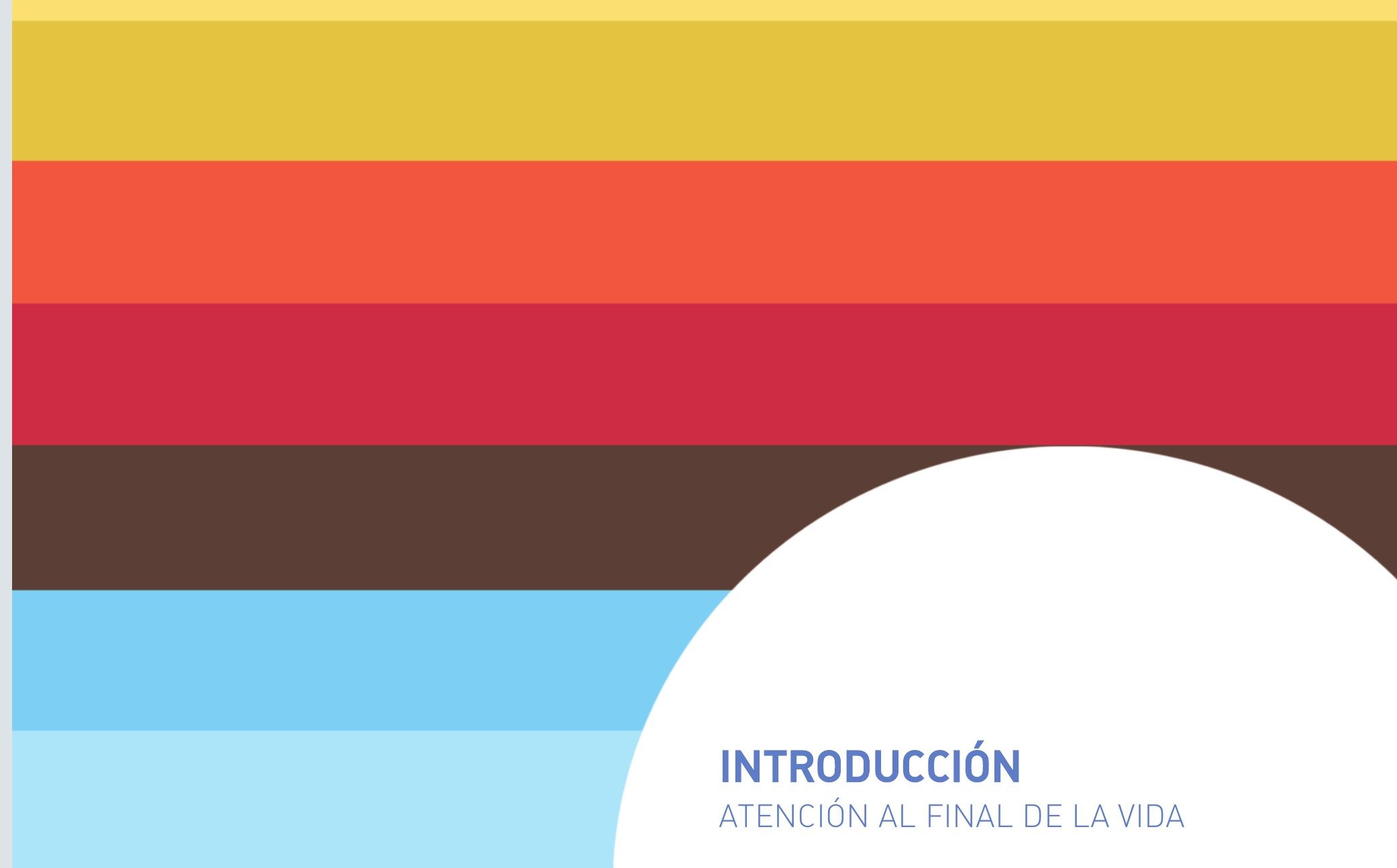


En países desarrollados, con tasas de envejecimiento entre un 15-20\% y cifras de mortalidad de $9-10 / 1.000$ hab, se estima que un $60 \%$ de
de una "situación de enfermedad avanzada y terminal".

En Extremadura, con una población aproximada de 1.100 .000 hab. y una mortalidad situación avanzada por año y, por tanto, con susceptibilidad de necesitar cuidados paliativos, se encuentra alirededor de 6.000 pacientes/año' (Gráfico 1) de los cuáles, unos 2.500 pacientes serán a causa de enfermedades oncológicas y un
cientes de enfermedades no oncológicas aproximadamente (Gráfico 2).

B.- Presencia de sintomas intensos, múltiples, multifactoriales y cambiantes con C.- Dependencia progresiva.

D.- Fuerte impacto emocional a pacientes, familias y equipos terapéuticos.

E- Altos niveles de necesidades $y$ demandas de cuidados.

F.- Evolución con frecuentes crisis de necesidades.

G.- Pronóstico de vida limitado. 
áREAS DESALD

HABTANTES

MORTALDAD GLOBA

No CÁNCRR ${ }^{3}$

\begin{tabular}{|l|c|c|}
\hline BADAJOZ & 267.481 & 2.407 \\
\hline MÉRIDA & 162.287 & 1.461 \\
\hline $\begin{array}{l}\text { DON BENIIO } \\
\text { VILLANUEVA }\end{array}$ & 141.929 & 1.277 \\
\hline LLERENA - ZAFRA & 106.762 & 980 \\
\hline CÁCERES & 197.201 & 1.775 \\
\hline CORIA & 47.516 & 428 \\
\hline PLASENCIA & 111.940 & 1.007 \\
\hline $\begin{array}{l}\text { NAVALMORAL } \\
\text { DELA MATA }\end{array}$ & 54.874 & 494 \\
\hline EXTREMADURA & 1.089 .990 & $\mathbf{9 . 8 1 0}$ \\
\hline
\end{tabular}

\begin{tabular}{|c|c|c|}
\hline 1.444 & 602 & 842 \\
\hline 877 & 365 & 512 \\
\hline 766 & 319 & 447 \\
\hline 588 & 245 & 343 \\
\hline 1.065 & 444 & 621 \\
\hline 257 & 107 & 150 \\
\hline 604 & 252 & 352 \\
\hline 296 & 123 & 173 \\
\hline $\mathbf{5 . 8 8 6}$ & $\mathbf{2 . 4 5 2}$ & $\mathbf{3 . 4 3 4}$ \\
\hline
\end{tabular}

- ÁREAS DE SALUD

PREVALENCA CÁNCER

PREVALENCIA NO CÁNCER

\begin{tabular}{|l|c|c|}
\hline BADAJOZ & 150 & 633 \\
\hline MÉRIDA & 91 & 385 \\
\hline $\begin{array}{l}\text { DON BENITO } \\
\text { VLLANUEVA }\end{array}$ & 80 & 336 \\
\hline LLERENA - ZAFRA & 60 & 253 \\
\hline CÁCERES & 111 & 467 \\
\hline CORIA & 27 & 113 \\
\hline PLASENCIA & 63 & 265 \\
\hline $\begin{array}{l}\text { NAVALMORAL } \\
\text { DE LA MATA }\end{array}$ & 31 & 130 \\
\hline EXTREMADURA & 613 & $\mathbf{2 . 5 8 2}$ \\
\hline
\end{tabular}

PREVALENCIA DE ENFERMOS TERMINALES

COBERTURA DIRECTA ÓPTIMA POR RECURSOS
ESPECIFICOS DE CUIDADOS PALLATIVOS

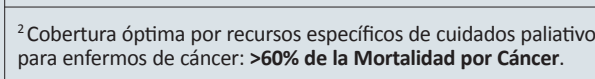

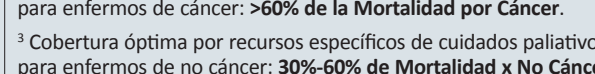

MÉTODO SIMPLIFICADO DE ESTIMACIÓN DE ENFERMOS TERMINALES Mortalidad anual enfermos terminales (enfermedades crónicas evolutivas)

6/1.000. Prevalencia enfermos terminales: $3 / 1.000$.
Escenario teórico para 1 millón de habitantes / mortalidad total de 9.000

Mortalidad:

Mortalidad Global $(9.000 /$ millón $=9 / 1.000)$
$60 \%(5.400 /$ millón $=5-6 / 1.000)$

$25 \%(2.250)$ cáncer
$35 \%(3.150)$ no cáncer

Proporción no cáncer $/$ cáncer $=1,5$ (hasta 2)

Prevalencia:

Cáncer (estimación supervivencia $3 \mathrm{~m}$ ): mortalidad x cáncer 2.250 / 4 = 560 No Cáncer (estimación media de supervivencia de 9 meses para no cáncer,
prevalencia $12 / 9=1,33):$ mortalidad $3.150 / 1,33=2.368$ Tasa Total $=2.290$ enfermos terminales $/$ millón $=2,9 / 1.000$ habitantes Proporción no cáncer $/$ cáncer $=4,2($ hasta $6-8$ )

Cobertura directa óptima de servicios de cuidados paliativos
( m millón yen función de la mortalidadl):

Cáncer: $>60 \%$ de mortalidad $=>1.350$
No Cáncer: $30-60 \%$ de mortalidad $=1.900-3.800$ 


\begin{tabular}{|c|c|c|c|c|c|c|c|c|c|}
\hline \multicolumn{9}{|c|}{ CónIGos UTILZZADOS DE LA CIE10 } & \multirow{3}{*}{$\begin{array}{r}\mathrm{gr} \\
\text { TOTAL }\end{array}$} \\
\hline \multicolumn{4}{|c|}{ 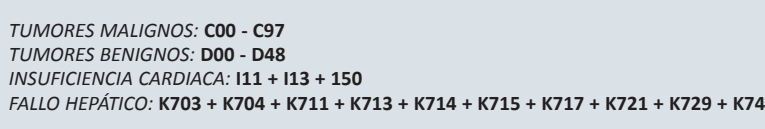 } & \multicolumn{5}{|c|}{ 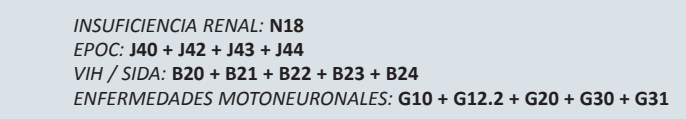 } & \\
\hline Ambos Sexos & $\begin{array}{l}\text { Tumores } \\
\text { Malignos }\end{array}$ & $\begin{array}{l}\begin{array}{l}\text { Tumores } \\
\text { Beningos }\end{array} \\
\text { a }\end{array}$ & $\begin{array}{l}\text { Insuficiencia } \\
\text { Cardiaca }\end{array}$ & $\begin{array}{c}\text { Fallo } \\
\text { Renal (IIC) }\end{array}$ & $\begin{array}{c}\text { Fallo } \\
\text { Hepatico }\end{array}$ & EPOC & $\begin{array}{c}\text { Enfermed. } \\
\text { Motoneuronales }\end{array}$ & VIH/SIDA & \\
\hline Andalucía & 15.808 & 591 & 4.547 & 600 & 1.100 & 2.336 & 2.332 & 259 & 27.473 \\
\hline Aragón & 3.341 & 137 & 828 & 207 & 119 & 397 & 507 & 35 & 5.571 \\
\hline Asturias & 3.552 & 59 & 649 & 109 & 149 & 475 & 497 & 35 & 5.525 \\
\hline Baleares & 1.980 & 51 & 578 & 103 & 95 & 208 & 292 & 50 & 3.357 \\
\hline Canarias & 3.697 & 103 & 567 & 127 & 201 & 384 & 389 & 67 & 5.535 \\
\hline Cantabria & 1.487 & 58 & 270 & 39 & 42 & 186 & 198 & 15 & 2.295 \\
\hline Castilla y León & 7.399 & 256 & 1.798 & 248 & 223 & 912 & 984 & 54 & 11.874 \\
\hline Castilla-La Mancha & 4.425 & 132 & 1.011 & 130 & 141 & 817 & 621 & 31 & 7.308 \\
\hline Cataluña & 15.976 & 569 & 3.607 & 698 & 754 & 2.349 & 3.012 & 138 & 27.103 \\
\hline Comunidad Valenciana & 10.288 & 331 & 2.319 & 437 & 566 & 1.426 & 1.696 & 154 & 17.217 \\
\hline Extremadura & 2.665 & 117 & 641 & 88 & 75 & 323 & 336 & 20 & 4.265 \\
\hline Galicia & 7.660 & 221 & 2.260 & 203 & 383 & 1.151 & 903 & 65 & 12.846 \\
\hline Madrid & 11.581 & 401 & 2.572 & 366 & 428 & 1.345 & 1.247 & 171 & 18.111 \\
\hline Murcia & 2.564 & 112 & 525 & 120 & 144 & 380 & 404 & 27 & 4.276 \\
\hline Navarra & 1.405 & 28 & 289 & 44 & 33 & 200 & 259 & 12 & 2.270 \\
\hline Pais Vasco & 5.653 & 157 & 938 & 188 & 263 & 669 & 868 & 52 & 8.788 \\
\hline La Rioja & 737 & 35 & 196 & 16 & 22 & 85 & 158 & 7 & 1.256 \\
\hline Ceuta & 124 & 6 & 25 & 5 & 7 & 16 & 17 & 4 & 204 \\
\hline Melilla & 97 & 7 & 26 & 6 & 6 & 16 & 12 & 3 & 173 \\
\hline TOTAL & 100.439 & 3.371 & 23.646 & 3.734 & 4.751 & 13.675 & 14.632 & 1.199 & 165.447 \\
\hline
\end{tabular}

Como población diana se incluyen²

A) Pacientes con enfermedad oncológica documentada, progresiva y avanzada con
escasa o nula posibilidad de respuesta a tratamiento espećfico y pronóstico de vida limitado.

B) Pacientes con enfermedad crónica evolutiva avanzada no oncológica, con limita-
ción funcional severa no reversible, con alto nivel de sufrimiento, cuyo pronóstico de

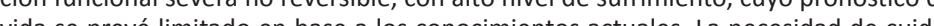
dos paliativos viene dada por el sufrimiento que provoca la presencia de sintomas in tensos, complejos, cambiantes o de complicaciones.

B.1. Pacientes con SIDA en los que exista una enfermedad documentada,
tivos.

B.2.- Enfermedades Respiratorias, como la enfermedad pulmonar obstructiva crónica o la fibrosis pulmonar en fases avanzadas, en la
que el maneio es áasicamente de soporte y el tratamiento complicaciones o reagudizaciones.

B.3. Enfermedades Cardiovasculares, como la insuficiencia cardíaca
de cualauier etiología y refractaria a tratamiento especifico, incluida cirugía y trasplante. B.4. - Hepatopatias Crónicas documentadas en fases avanzadas sin
posibilidad de trasplante ni tratamientos especificos. a

B.5. Enfermedades Neurológicas, como las demencias en progresión
y fase muy avanzadara enfermedad de Parkinson cas en y fase muy avanzada, entermedad de Parkinson avanzada, Esclero-
sis Lateral Amiotrófica y otras enfermedades neurodegenerativas que no se beneficien de tratamiento específico.

B.6.- Enfermedades Renales que provoquen una insuficiencia renal
crónica sin posibilidad de diálisis $y /$ o trasplante renal.

MORTALIDAD POR CAUSA Y COMUNIDAD AUTÓNOMA (ESPAÑA, 2008) 


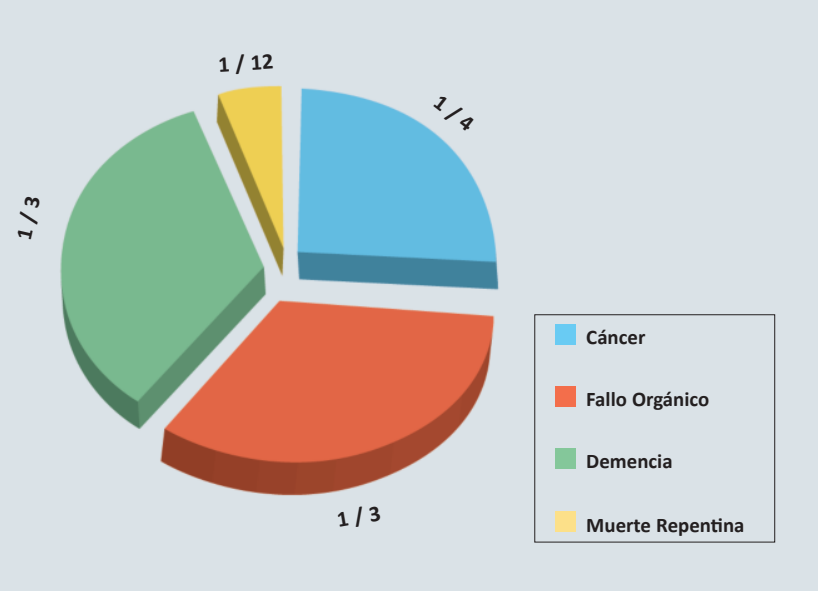

TRAYECTORIA DE LA DEMENCIA.
DEL DIAGNOSTICOA ALA MUERTE

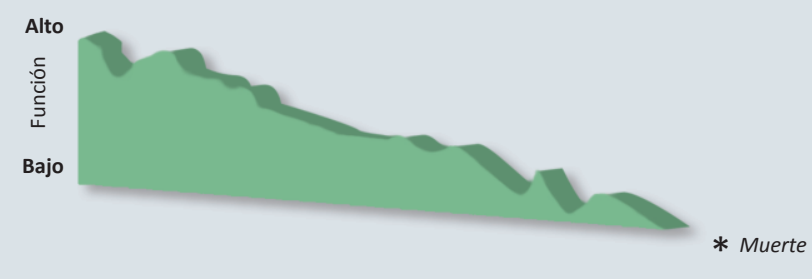

Deterioro lento y progresivo que puede durar $6-8$ años

PATRONES EVOLUTIVOS DE LA ENFERMEDAD AVANZADA Y TERMINA

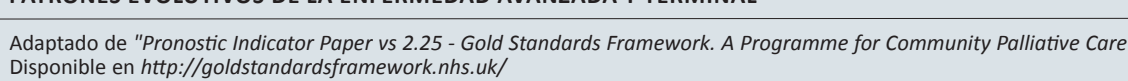

TRAYECTORIA DEL CÁNCER.
DEL DIAGNOSTIICO A LA MUER Alto
$\frac{\bar{g}}{\overline{\frac{5}{4}}}$
Bajo

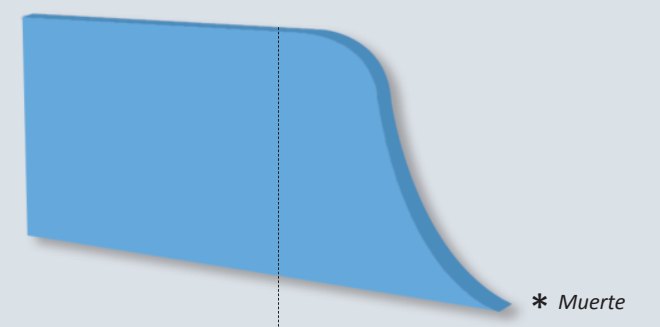

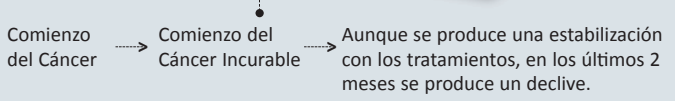

PATRONES EVOLUTIVOS DE LA
ENFERMEDAD AVANZADA Y TERMINAL

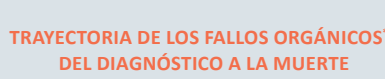



la muerte de forma repentina e inesperarada

Según la Organización Mundial de la Salud (OMS) los Cuidados Paliativos (CP) pueden
definirse como aquellos que mejoran la calidad de vida del paciente y sus familias frente a problemas assciados a situaciones de enfermedad avanzada y terminal, evaluación impecable y y liviot del sufrimiento, mediante la identificación precoz, dolor y otros problemas físicos, psico-so-
ciales y espirituales. Entre otras cuestiones, los cuidados paliativos:

- Proporcionan alivio frente al dolor $y$ otros sintomas.

Reafirman la vida y consideran la muerte como un proceso normal, evitando posponerla.

Integran los aspectos psico-sociales y espirituales en el cuidado. 

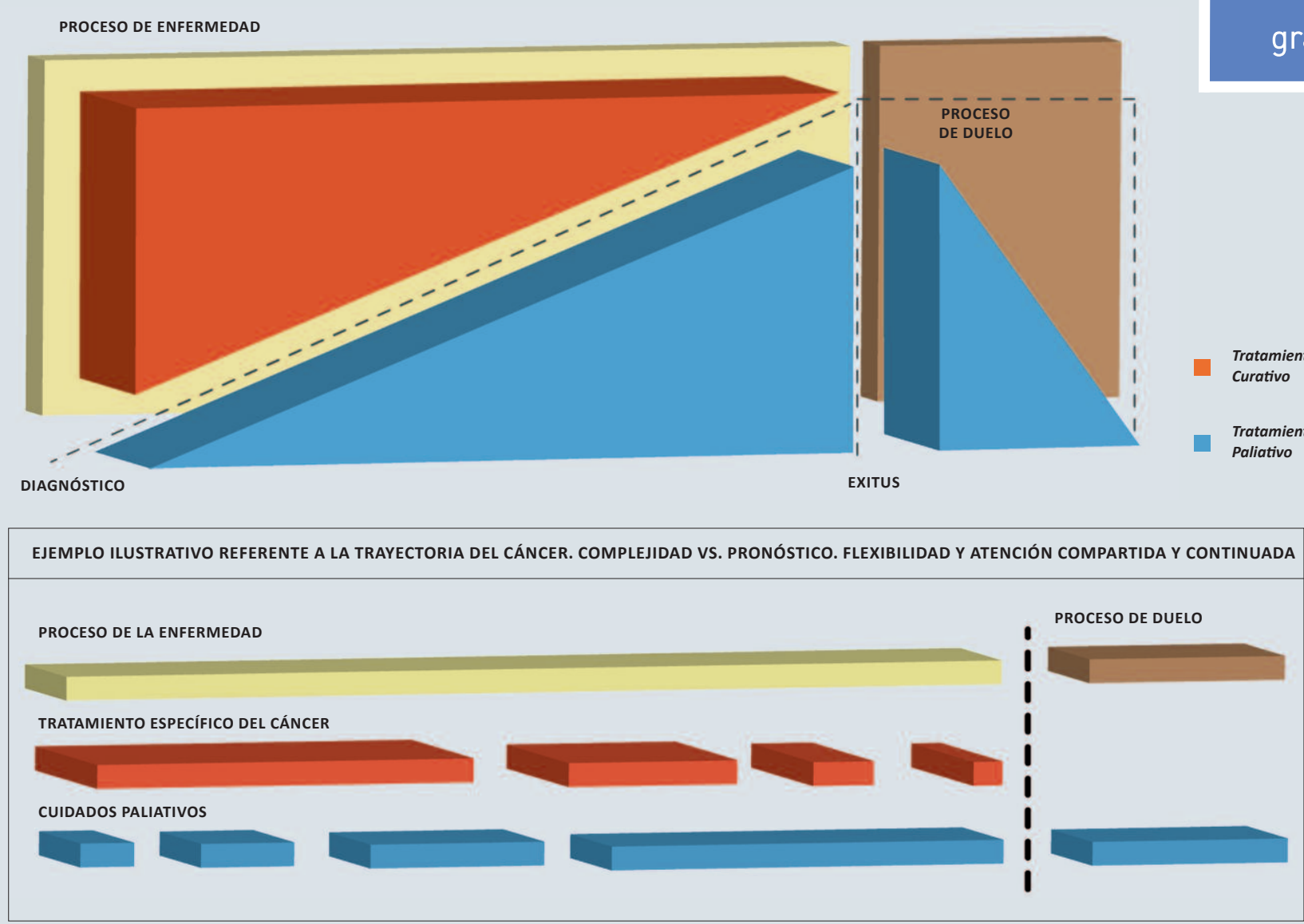

- Ofrecen un sistema de soporte para los familiares durante la enfermedad y el proceso de duelo. - Utilizan una aproximación interdisciplinar para el conocimiento de las nece-
sidades de los pacientes y sus familiares.

- Mejoran la calidad de vida y pueden incluso influir positivamente sobre el

- Son aplicables de forma precoza durante la enfermedad al mismo tiempo que
otras terapias. La intervención específica y paliativa puede convivir simultáneamente, dependiendo de la intensidad de cada intervención y en función de la complejidad de la situación y del pronóstico, así como del establecimiento de una verdadera atención compartida y continuada entre todos los profesionales
que intervienen en el proceso de la enfermedad (Gráfico 4).

En España los Cuidados Paliativos son una prestación sanitaria básica y un dere

En el año 2001, el Ministerio de Sanidad y Consumo publicó el Plan Nacional de cientes en situación terminal a la asistencia en cualquier lugar, circunstancia o stuación.

En Marzo de 2007 y enmarcado dentro del Plan de Calidad del Sistema Nacional de Salud se aprueba la Estrategia Nacional en Cuidados Paliativos con el objetivo
de consensuar entre todas las Comunidades Autónomas líneas estratégicas que mejoren la atención paliativa en nuestro país 


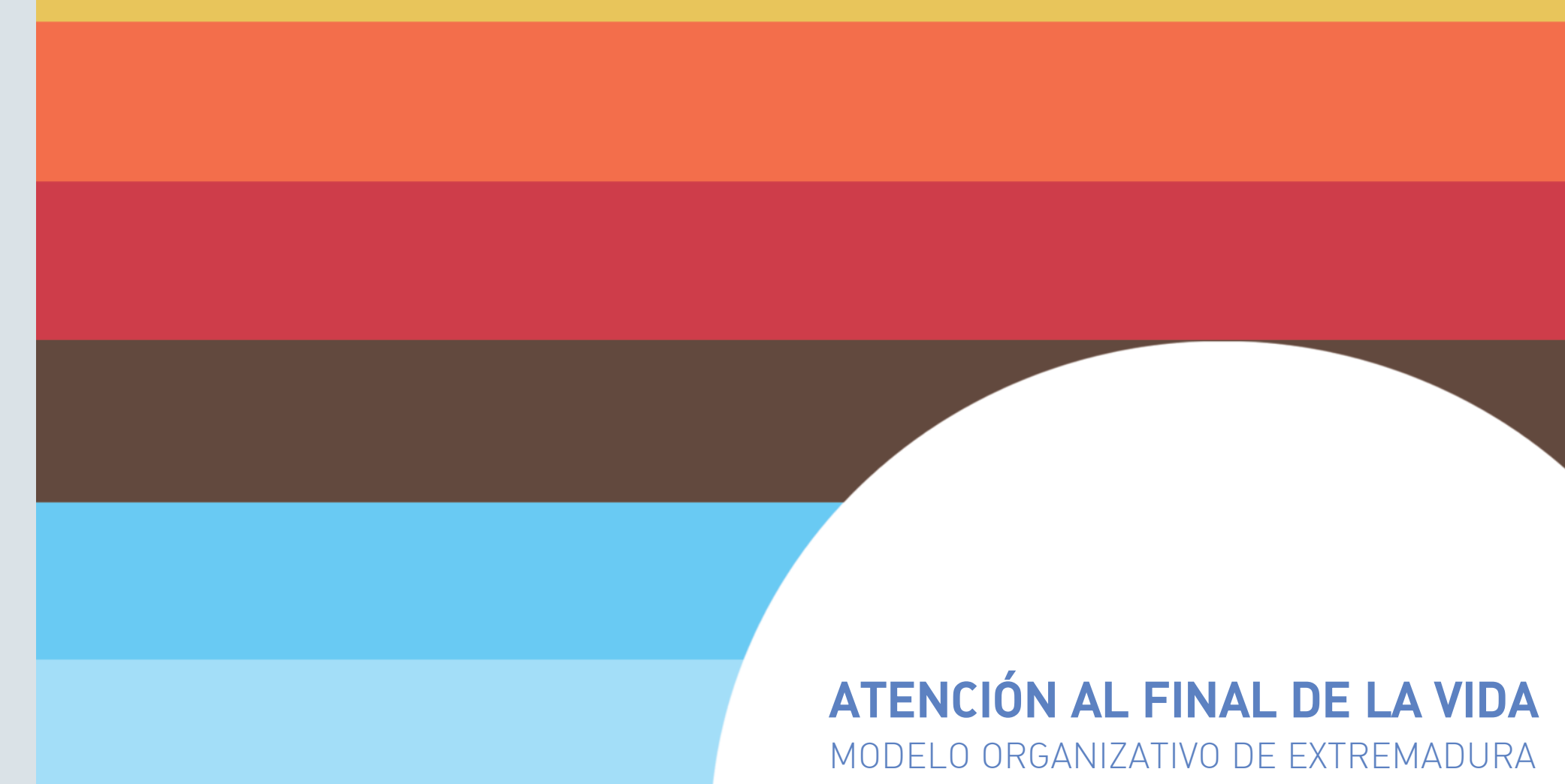


UNIDADES DE

CUIDADOS PALIATIVOS

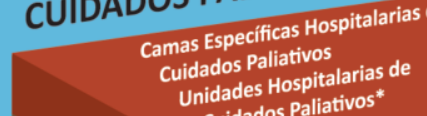

EQUIPOS DE SOPORTE DE CUIDADOS PALIATIVOS

RECURSOS GENERALES

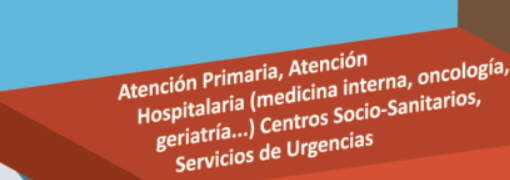

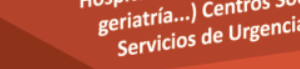

MODELO ORGANIZATIVO
DE EXTREMADURA

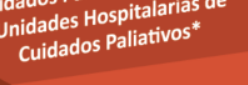

\section{de}

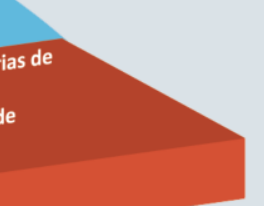

El Programa Regional de Cuidados Paliativos de Extremadura (PRCPEx) nace, en el
año 2002, con el compromiso de establecer un conjunto sistemático de medidss, forma progresiva, encaminadas a mejorar la atención al final de la vida dentro de nuestra Comunidad Autónoma.

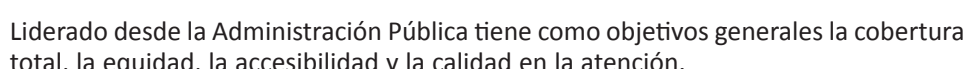

Entre sus caracteristicas destacan:

1.-Un modelo de atención basado en "necesidades de pacientes y familias" (ATENcIón INTEGRAL) como un proceso sistemático que tiene en cuenta todas las necesi-

2.- Un modelo de organización basado en: (ATENCIÓN INTEGRADA, COMPARTIDAY
CONTINUADA)

2.1 - Atención Integrada y compartida entre todos los niveles asistenciales con
intervenciones basadas en la complejidad que garantice la continuiddd asisten intervenciones basadas
cial (Gráfico 5, Gráfico 6 )

2.2- "Atención Continuada”: 24h/365d Recursos de Urgencias Generales: (Puntos de Atención Continuada en
Atención Primaria (PAC), Servicios de Emergencias (112), Servicios de

Recursos de Urgencias Especifico de Cuidados Paliativos: (Servicio de Asesoramiento Teletonico
de Soporte de Cuidados Paliativos de Extremadura (ESCP). (Grófico 7 ) 
a) Caracteristicas personales

a1. Niños / Adolessentes / Jóvenes / Adultos

Trabstornas de adicción (alcohol, drogas .

a. Escasa red de apoyv socia
b. Claudicación familiar

(

d. Familias con dueles
e. Hostilidad familiar

b) Situaciones Clinicas Complejas

b1. Sintomas de difícil control (EVA >6) o sintomas refractarios

13. Sintomas refractarios que requieren sedación paliativa o que precisen

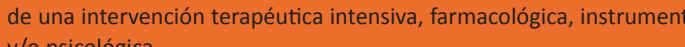

b4. Obstrucción intestinal / urológica

W5. Situacion de ultimos dias con insuficiente control

17. Transtornos psico-emo-

Transtornos adaptativos severos

.

c) Situaciones Clinicas de deterioro rápido con crisis de

c.. Úlceras tumorales, fistulas $u$ otras lesiones de piel y mucosas que re

DEPENDIENTES DE LOS PROFESIONALES

a. Conocimiento y/o motivación insuficiente sobre el b. Dilemas éticos y de valores, actitudes $y$ creencias c. Profesionales referentes con duelo propio no resuelto o con otros equipos respecto a la toma de decisiones

ESTRATEGIA DE ACTUACIÓN COMPLEJA

a. Rotación de opioides
b. Indicacicón y Gestión de tratamientos especistes

Radicacion y Gestión de tratamientos especiales:
Radioterapia, Gastrostomia endoscópica, técnicas

analgésicas invasivas,...
c. Demanda reiterada de eutanasia o suicicio asistido

c. Endicación y anlicación de sedación paliativa

d. Indicación y aplicación de sedación paliativa
e. En domicilio, indicación y manejo de hidratación,

nutricíon enteral, parenteral, paracentesis, curas
de difíil manejo
OBJETIVOS

3.1- Cobertura tanto de pacientes oncológicos como no oncológicos.
3.2. Accesibilidad.

4- calidad: eficacia, eficiencia y efectividad.

EVALUACIÓN 4.- Un modelo de organización que realice una "evaluación sistemática de resultados" con el objetivo de realizar una planificación periódica ajustada a necesidades asistenciales, formativas e investigadoras
5.1. - La "Formación planificada y adaptada" a todos y cada uno de los colectivos,
que de alguna u otra forma, intervienen en todo el proceso: 


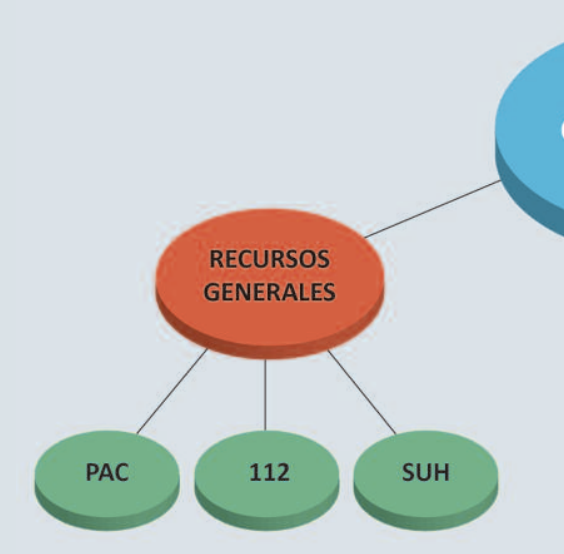

ATENCIÓN

CONTINUADA $Y$
DE URGENCIA

DE URGENCIA

DE URGENCIA

RECURSOS

ESPECiFICOS

ASESORAMIENTO
TELEFÓNICO POR EL ESCP

El objetivo general deI
Asserormiento lefforico es
offecer una continuidad asis-

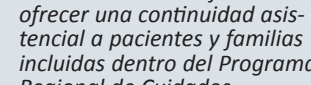

Incluidas dentro del Program
Regional de Cuidodos
Paliativos (PRCPCX), mediante

soporte telefónico por los
Profesionales de Cuidados

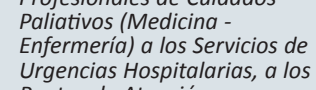

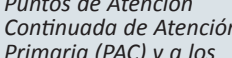

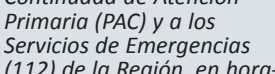

PROTOCOLO DE ATENCIÓN

1 SITUACIÓN

VALORACIÓN

situación de urgencias en la
evolución de la enfermedad
and

evolucion ne la entermedaca de
un paciente en fase avanzada o
tetrminal que se encuentra en
domicilio

4 SOLuCión

$\begin{array}{ll}\text { debe acudir o avisar a los } & \text { el equipo sanitario, y siempre } \\ \text { profesionales del Punto de } \\ \text { Atención Continuada que le }\end{array} \quad \begin{aligned} & \text { segun su criterio, valorará } \\ & \text { contactar con el Médico o }\end{aligned}$

$\begin{array}{ll}\text { Atención Continuada que le } & \text { contactar con el Médicoo } \\ \text { corresponda según su Area de } & \text { Enfermera de Cuidados }\end{array}$

$\begin{array}{ll}\begin{array}{l}\text { Salud (en caso de emergencia } \\ \text { al so 112). }\end{array} & \begin{array}{l}\text { Paliativos según provinci } \\ \text { (Cáceres o Badajoz) }\end{array} \\ & \end{array}$

Ecibidial a i información, el
Medico o Enfermera de Cuidados Paliativos realizarc
un seseremeniento sche las dinar recurusos entre difrents
niveles asistenciales a) Profesionales de la Salud en general

b) Profesionales con mayor prevalencia en la atención de la población diana
(Equipos de Atención Primaria, Servicios de Oncologia Médica, Servicios de erriatriá, Servicios de Medicina Interna, Servicios de Urgencias en Atención Centros Socio-Sanitarios (CSS) o Centros de Atención a la Dependencia (CAD). c) Profesionales de Recursos Especificos de Cuidados Paliativos d) Voluntariado

e) Familiares y Cuidadores

f) Población General

5.2- La "Investigación" como forma de generar más y mejor evidencia científica. 5.3- Una estructura de "trabajo en red", integrada por todos los profesionales de 
MARIA MEDICINA INTERNA
ONCOLOGÍA GERIATRIÁA CENTROS SOCIO

UNTARIADO ESCP ATENCION PRIAS VO

MEDICINA INTERNA ONCOLOGÍA GERIA
TRIA CENTROS SOCIO SANITARIOS SERVI

TRIA CENTROS SOCIO SANITARIOS SERVI
CIOS DE URGENCIAS VOLUNTARIADO ESCP

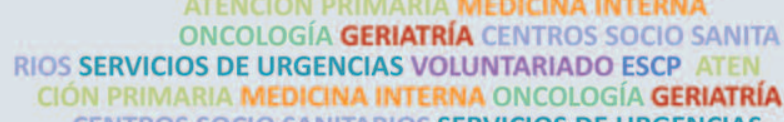

CENTROS SOCIO SANITARIOS SERVIICIOS DE URGENCIAS

VOUUNTAAIADOESCP AENCEON PRIMARIA MEDICANA INTER

DE URGENCIAS VOLUNTARIADO ESCP ATENCIÓN PRIMARIA MEDI

CINA INTERNA ONCOLOGIAA GERIATRRIA CENTROS SOCIO SANITA
RIOS SERVICIOS DE URGENCIAS VOLUNTARIADO ESCP ATEN

TRIA CENTROS SOCIO SANITARIOS SERVICIOS DE UR

GENCIAS VOIUNTARIADO ESCP OTENCION PRIMARIA

MEDICNA INTERNA ONCLLLOGIA GERRATRÍA CENTROS
SOCIO SANITARIOS SERVICIOS DE URGENCIAS VOLUNTA

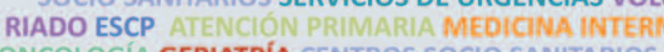

ONCOLOGIA GERIATRÍA CENTROS SOCIO SANITARI
SERVICIOS DE URGENCIAS VOUUNTARIADO ESCP

TIENCIÓN PRITARIA MEDICINA INTERNA ONCC

SEVICIOS DE URGENCIAS VOLUNTARIADO

ESCP ATENCION PRIIMARIA MEDICINA
INTERNA SERVICIOS DE URGENCIAS ESCP

$$
\begin{aligned}
& \text { ESCP } \\
& \text { SOCIO SANITARIOS }
\end{aligned}
$$




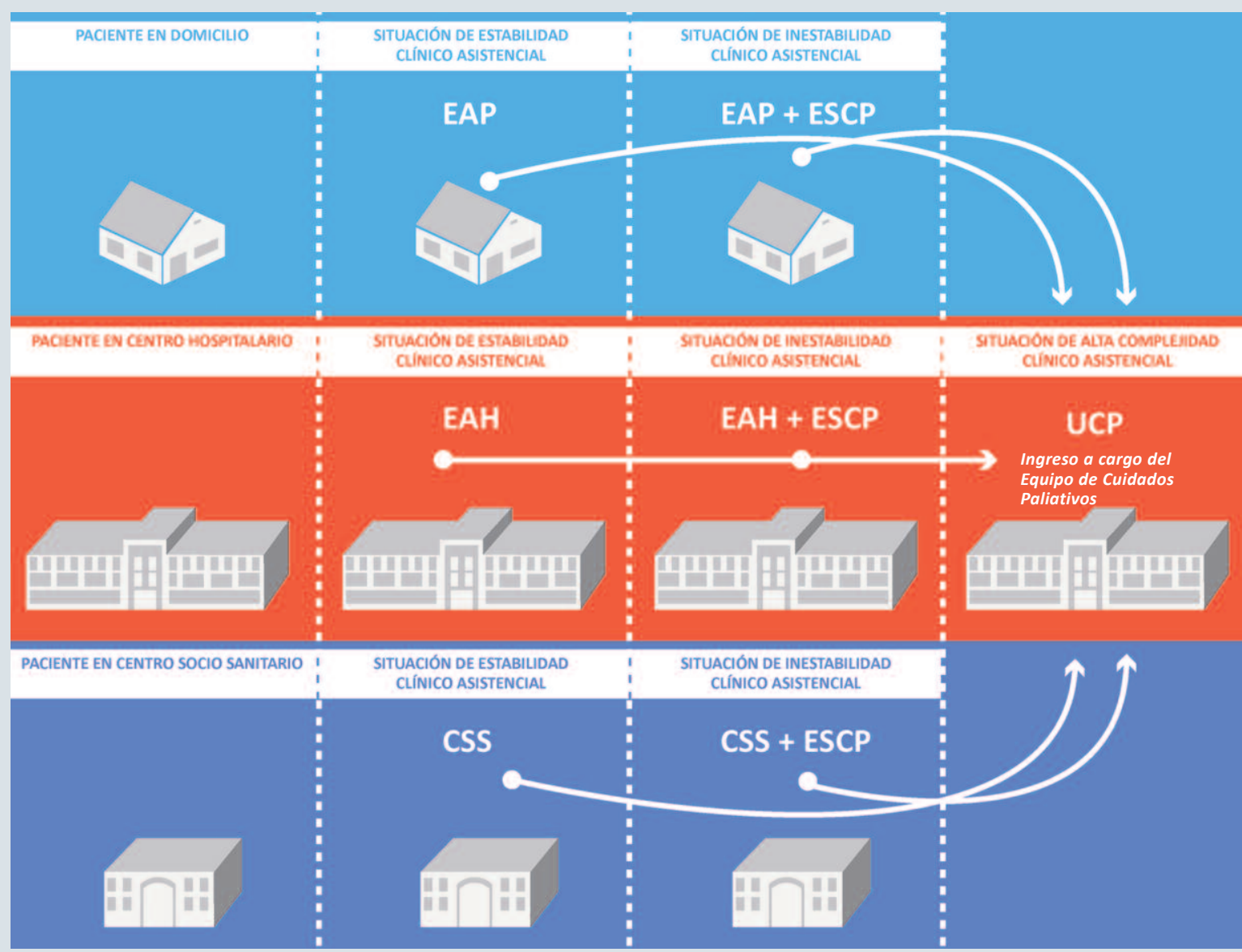

La atención al final de la vida en nuestra Comunidad Autónoma se organiza de la
siguiente manera (Gróf vico g)

1.- Atención Primaria: los Profesionales de Atención Primaria realizan la atención paliativa cuando el paciente se encuentre en su domicilio.

2.- Servicios Hospitalarios: con alta prevalencia de pacientes en situación de en-
fermedad avanzada y terminal. Los profesionales de estos servicios realizan Ifermedad avanzada y terminal. Los profesionales de estos serve
atención paliativa de los pacientes durante el ingreso hospitalario. 3.- Centros Socio-Sanitarios o de Media-Larga Estancia: Los profesionales que tra-
bajan en estos centros realizan la atención paliativa de los pacientes residentes en
ellos bajan
ellos.

MEDIDAS Las medidas en los servicios generales son aquellas actuaciones que se ponen en marcha, en servicios con prevalencias especialmente altas de pacientes
dades avanzadas y terminales, para mejorar la calidad en la atención.

Entre ellas se encuentran:

A.- Medidas para mejorar la cobertura de atención a pacientes con necesida-
des de recibir cuidados paliativos dados paliativos

Establecimiento de criterios de identificación de pacientes en situación de enferB.- Medidas para mejorar la accesibilidad a pacientes $y$ familias

Establecimiento de circuitos de coordinación entre recursos generales y especif-
cos de cuidados paliativos.

ORGANIZACIÓN DE LA ASISTENCIA EN FUNCIÓN DE SUS NIVELES DE COMPLEIDAD

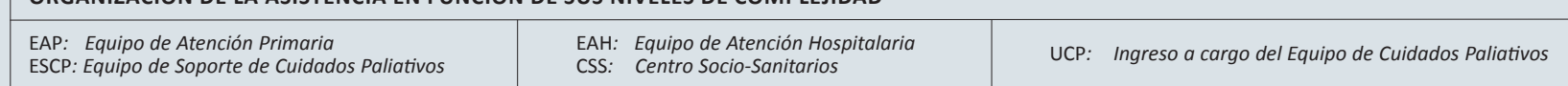


Valoración, desde el equipo responsable, de cuál sería la respuesta a la pregunta: ¿Le sorprendería que este pa-
ciente falleciera en los próximos $6-12$ meses?

ELECCIÓN / NECESIDAD

2 El paciente con una enfermedad avanzada y terminal puede elegir si desea recibir cuidados paliativos

\section{3 mocoseres anmeses}

3 Indicadores especificos para cada uno de los tres tipos principales de grupos de enfermedad al final de la vida:

la valoración integral de cada paciente nos ayudará a ver globalmentet la situación del mismo, asi como personalizar las decisiones, que deben estar
basadas en el juicio clinico en función de los factores pronósticos citados, y también en las opiniones y deseos del paciente, de la familia y del equipo basadas en eljucto
multidiscplinar.

CRITERIOS DE IDENTIFICACIÓN DE PACIENTES EN SITUACIÓN DE ENFERMEDAD AVANZADA Y TERMINAL

Reuniones interequipos, sesiones de casos clínicos, elaboración de protocolos conjuntos...

E.- Medidas encaminadas a mejorar el registro y documentación clínica

Desarrollo de la historia clínica ́única informatizada con registros de síntomas y
plan de tratamientos y cuidados (JARA). F.- Medidas para mejorar la competencia y la capacidad de resolución de los profesionales Formación especifica en materias relacionadas con los cuidados paliativos (con-
trol sintomático, habilidades de comunicación, bioética aplicada a la toma de detrol sintomatico, habilidades de comunicación, bióética aplicada a la toma de de-
cisiones al final de la vida, trabajo en equipo, apoyo a la familia, atención al due- 


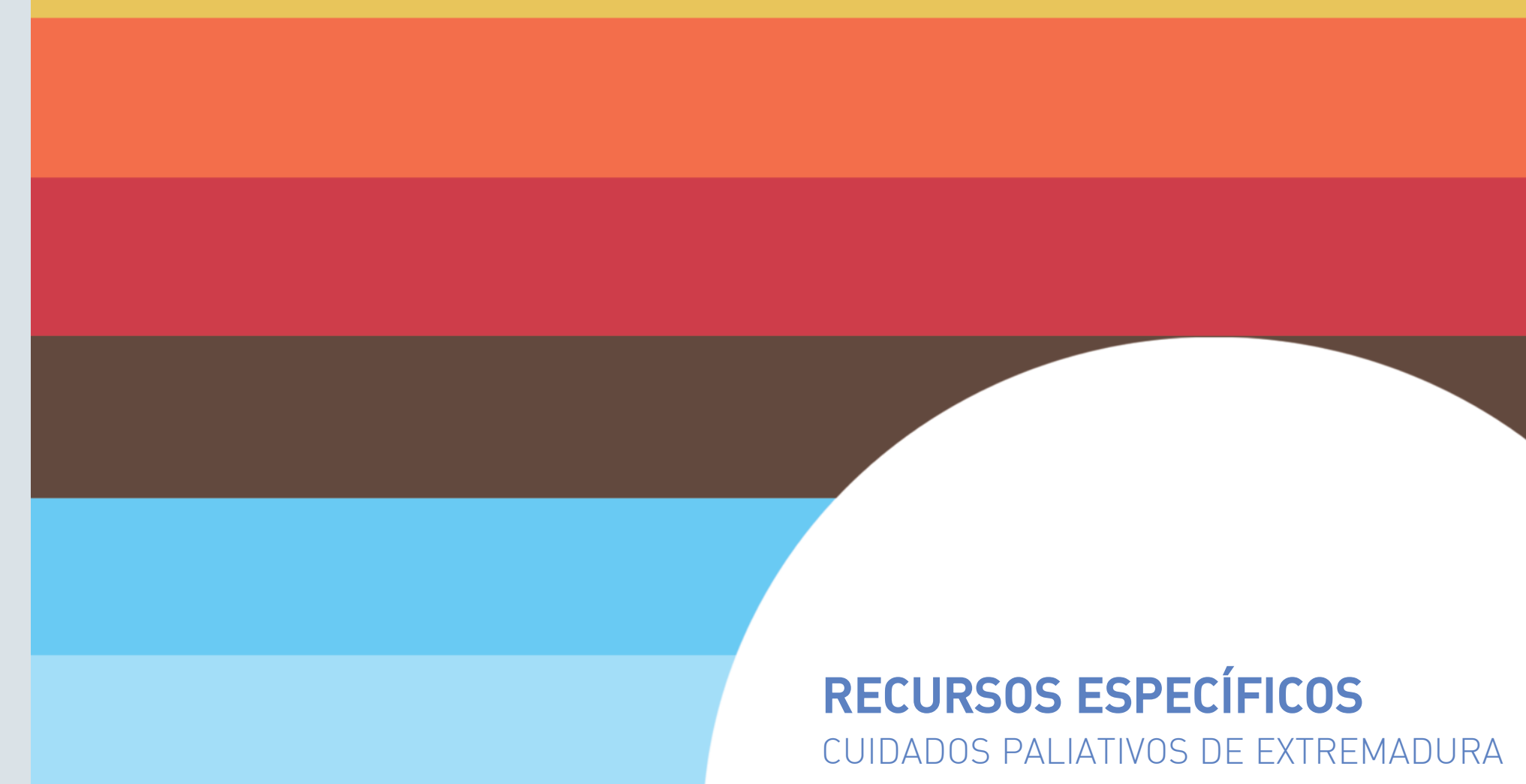


CONSEJERÍA DE

SANIDAD Y DEPENDENCIA

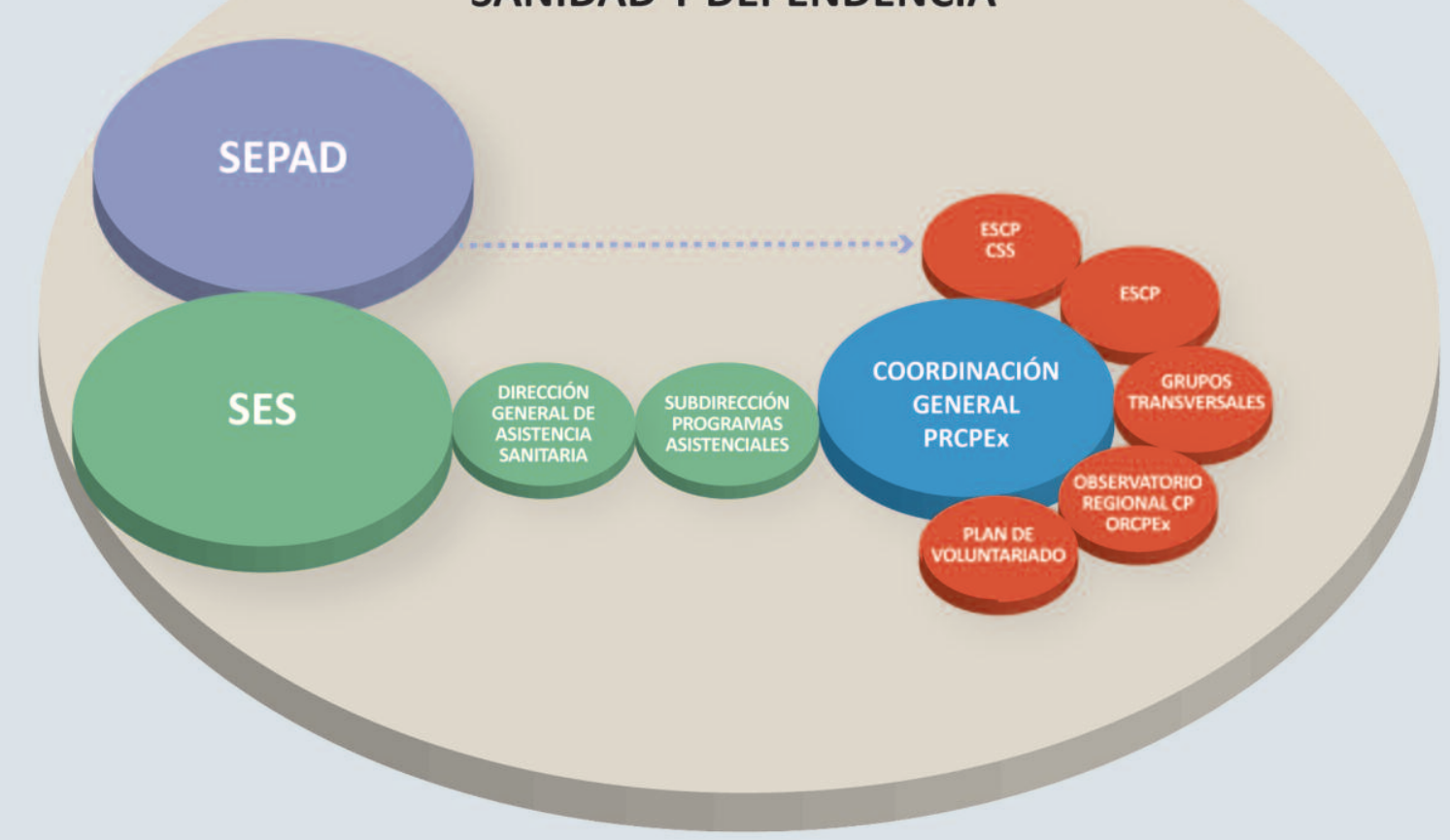

- Coordinar l a actividad asistencial, formativa e investigadora de los profesionales

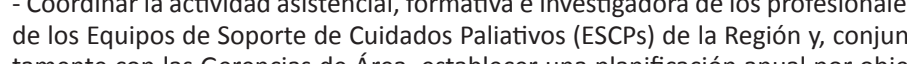
tamente con las Gerencias de Area, establecer una planificacion anual por obje-

- Apoyar e incentivar los "grupos de trabajo" " "Eormación", "Registro ción" ff formado por professionales de los ESCP, estableciendo planes anuales de trabajo bajo criterios de calidad

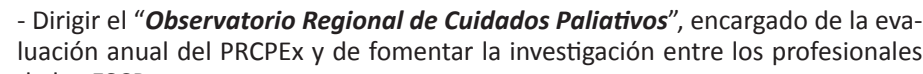

- Potenciar el "Plan de Voluntariado de Cuidados Paliativos" y organizar, junto planificación anual del trabajo guiada por criterios de calidad.

Establecer relaciones profesionales, tanto a nivel nacional como internacional, con otros estamentos relacionados con los cuidados paliativos así como institucon otros estamentos relacionados con los cuidados paliativos asi como institu-
ciones universitarias, sociedades cientificas, fundaciones, colegios profesiona-
les. - Tener " "isión de futuro" e implementar "estrategias innovadoras" en cuánto al
desarrollo de la atención al final de la vida dentro de nuestra Comunidad 


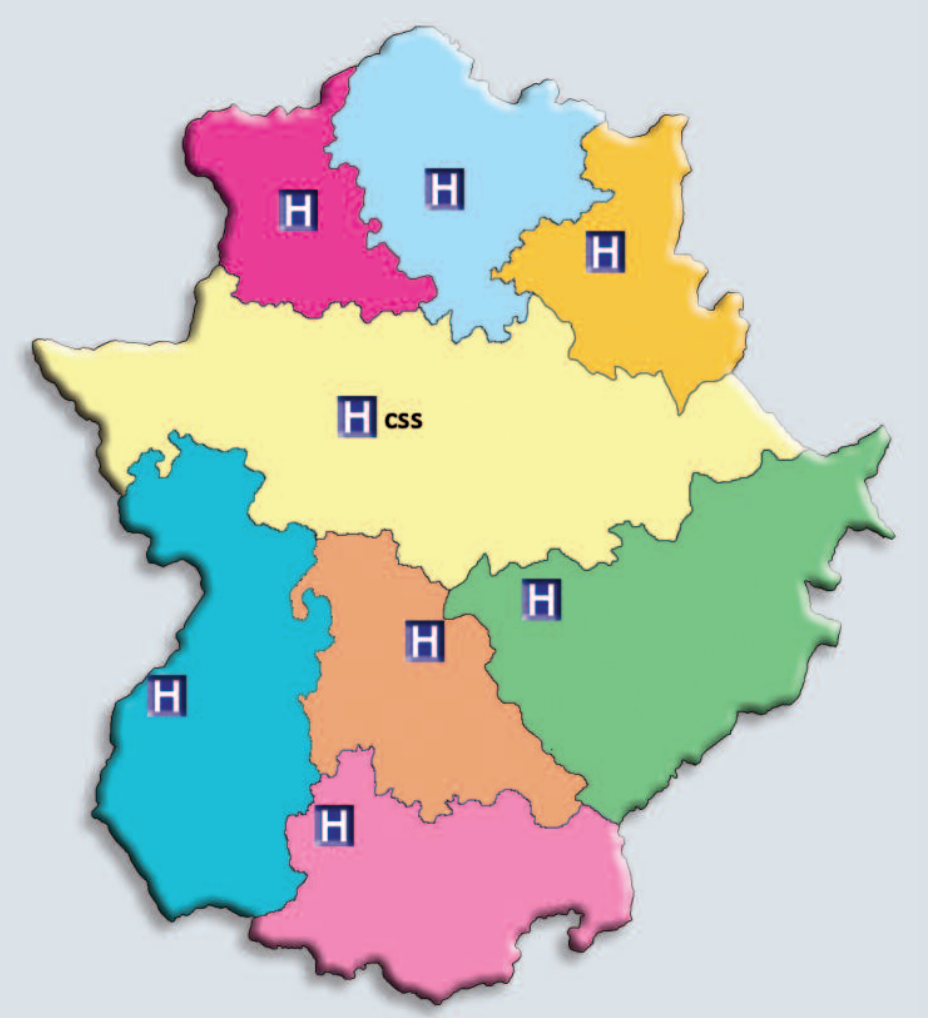

Los Profesionales que integran los Equipos de Soporte de Cuidados Paliativos (ESCPs),
realizan una atención compartida con todos los profesionales de los diferentes piverealizan una atención compartida con todos los profesionales de los diferentes nive
les asistenciales, basando la intensidad de su atención, en la compleijidad de la situación a atender y en la coordinación con los profesionales con los que comparten la atención. Existe un ESCP en cada Area de Salud (Grafico 12), integrado por médicos, como hospitalario. Recientemente se ha incorporado un nuevo forma especifica, en el soporte a profesionales de varios centros socio-sanitarios de

Los Niveles de Intervención de un Equipo de Soporte de Cuidados Paliativos son:

COMPLEO HOSPITALARIO DE BADAJC COMPREO HOSPTTALARI DE CACERES
CENTROS SOCIO SANITARIIOS DE CACERES

HOSPITAL DE MÉRIDA

HOSPTTAL “VIRGEN DEL PUERTO” DE PLASENCA

- HOSPTTAL DON BENITO - VILAAUEEUA

hospittal "IIUDAd de CORIA"

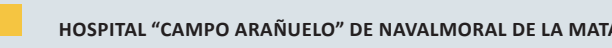

HOSPTTLL DE ZaFRA- LLRENA

1.- Asesoramiento puntual, directo o telefónico.

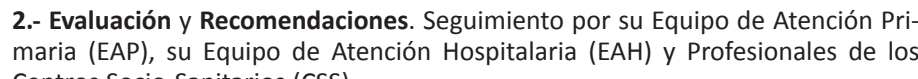

3.- Evaluación y Seguimiento compartido con intervenciones intermitentes del Atención Primaria (EAP) Equipo de Atención Hospitalaria (EAH) y de Profesionales de los Centros Socio-Sanitarios (CSS).

4.- Evaluación y Seguimiento continuo y compartido entre el Equipo de Soporte de Cuidados Paliativos (ESCP) y el Equipo de Atención Primaria (EAP),
Equipo de Atención Hospitalaria (EAH) y los Profesionales de los Centros 


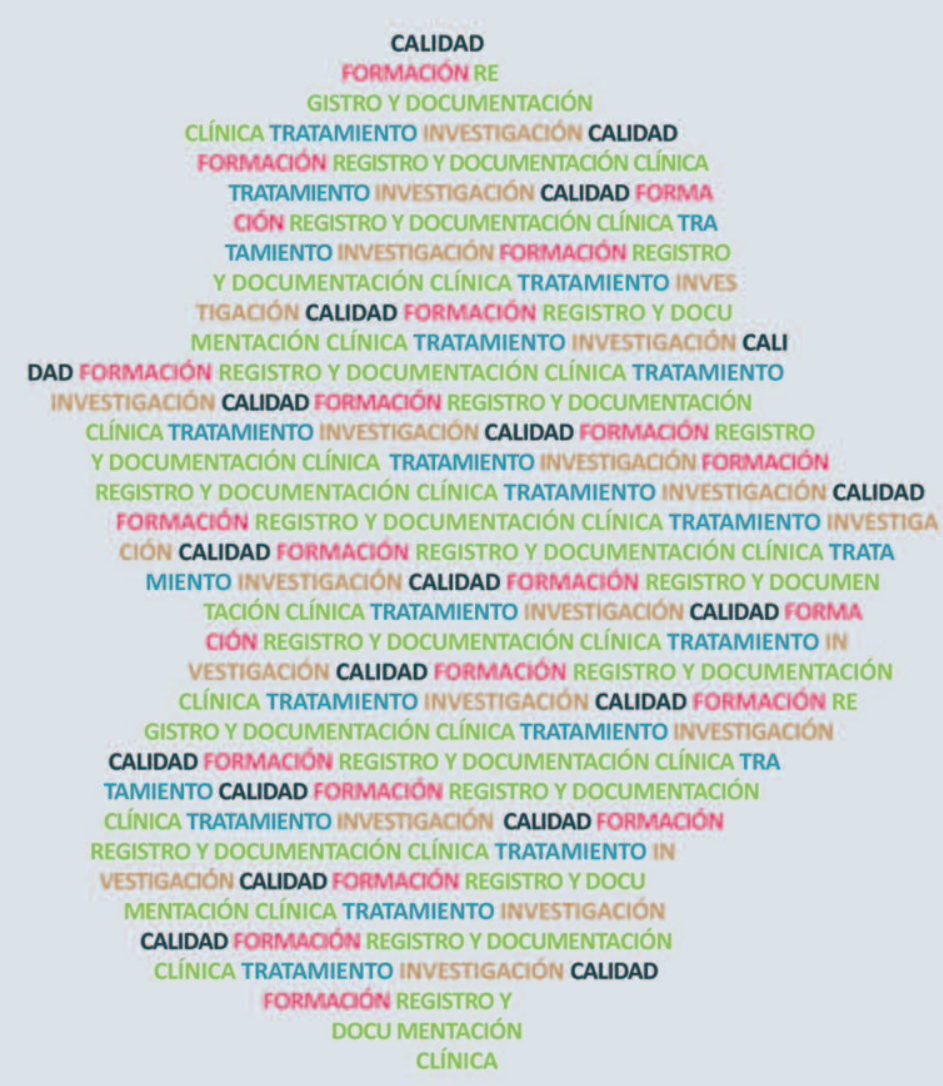

Dentro del PRCPEx existe una red de trabajo (Gráfico 13) donde participan activamente los profesionales de cuidados paliativos de todas las áreas de salud. Cada gru-
po lo forman un coordinador de grupo y un secretario. Mediante una planificación
anual desarrollan un programa con:

Objetivos a realizar durante el año

Evaluación con planes de mejora.
Memoria Anual.

Los Grupos de Trabajo establecidos son

Formación (FORPALEX): Tiene como objetivo fundamental gestionar la actividad docente en cuidados paliativos a realizar cada año. Para ello desarrolla un plan anual de formación basada en las necesidades de los profesionales del Servicio Fundación para la formación e Investigación de los Profesionales de la Salud de Extremadura (FUNDESALUD), principalmente.

Registro y Documentación Clínica (REGPALEX): Tiene como objetivo planificar la
recogida de información de los ESCPS. Colabora en el diseño y mantenimiento de recogida de información de los ESCPs. Colabora en el diseño mantenimiento de medidas que mejoren el fluio de información entre niveles asistencives, ascico mo la adecuada custodia de los datos clínicos. Asimismo, vela por la correcta recogida de los datos que se precisan para la realización de investigaciones mult-
centricas.

Tratamientos (TRAPALEX): Como misión principal se encuentra asegurar el con-
tacto de los profesionales con la mejor y más actual evidencia cientifica en cuipacto en la materia y fomenta la revisión de artículos de especial relevancia. Además, participa en la elaboración de protocolos y yuías clinicas para su difu-
sión entre los profesionales sanitarios del Servicio Extremeño de Salud 
CONSEJERÍA DE

SANIDAD Y DEPENDENCIA
SES

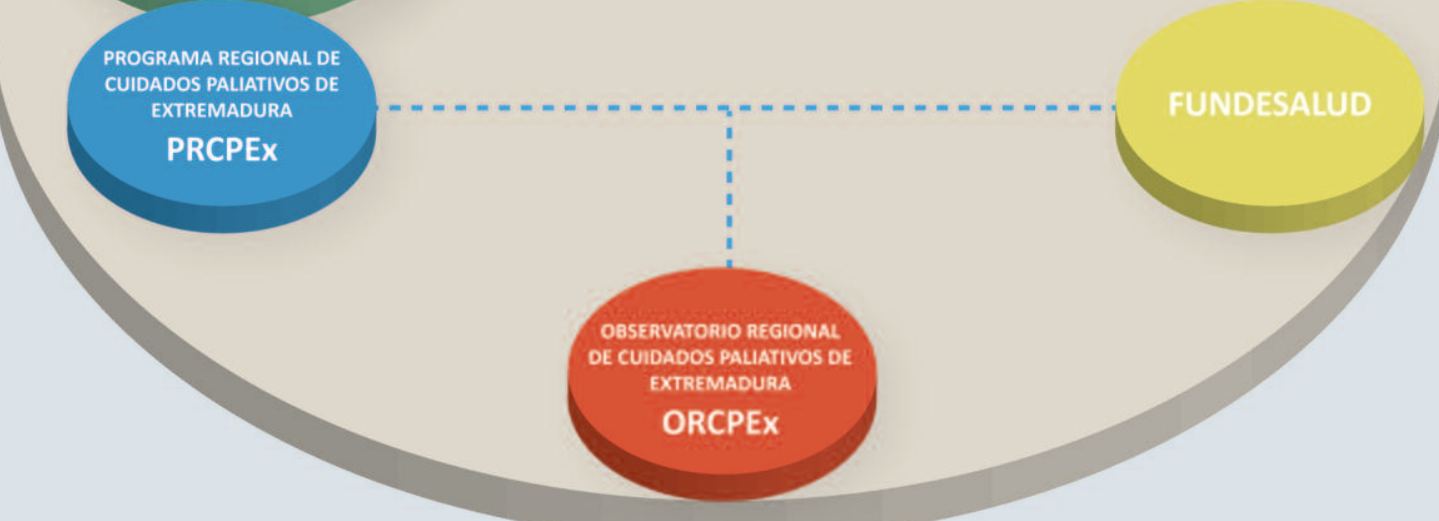

OBSERVATORIO REGINAL
DECUIDADOS PALATINOS
Calidad (CALPALEX): Se encarga de diseñar estrategias de mejora en la calidad de to de los mismos, estableciendo planes de mejora anualmente.

Investigación (IPALEX): Tiene como objetivo fomentar la investigación de los pro-
fesionales de los ESCPS y compartiri esos conocimientos con la comunidad cientifesionales de los ts
fica en general.

Observatorio Regional de Cuidados Paliativos de Extremadura (ORCPEX), se crea en el año 2006, con el objetivo de gestionar el conocimiento en el ámbito de los cuida El Observatorio (ORCPEx) desarrolla su labor en torno a 4 grandes lineas estratégicas. 1.- Evaluación: desarrollo de estudios que sirvan de base a la toma de decisiones
en la gestión del Programa Regional de Cuidadas Paliativos de Extremadura. 2.- Investigación: soporte a la investigación que se desarrolla en el PRCPEx, esta-
blecimiento de relaciones y colaboraciones con otros observatorios o grupos de investigadores.

3. Formación: diseño de planes de formación en investigación.

4.- Difusión: creación de sistemas de difusión de la información y de la produc-
ción cientifica en el ámbito de los cuidadas paliativos. cion cientifica en el àmbito de los cuidados paliativos.

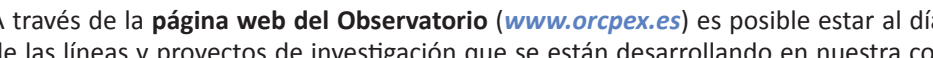
de las ineas y proyectos de investigación que se están desarrollando en nuestra comunidad, conocer la oferta formativa y beneficiarse de los recursos web disponibles
(foro, biblioteca de cuidddos paliativos, noticias, enlaces, 
Pastoral de la Salud de la Diócesis de Coria-Cáceres

Asociación Parkinson Extremadura

Asociación de Familiares y Pacientes de Alzheimer de Mérida

Asociación Extremeña de Laringectomizados

Pastoral de la Salud de la Diócesis de Plasencia

Asociación de Voluntarios Mayores de Mérida

Red de Voluntariado de Guareñ

Asociación Oncológica "Tierra de Barros"

Fundación Ícaro

B.- Seleccionar y formar voluntarios especificamente en cuidados paliativos. C.- Establecer circuitos de coordinación entre diferentes Entidades de Voluntacon los cuidados paliativos.

El Plan de Voluntariado en Cuidados Paliativos de Extremad
compuesto por 14 Entidades sin ánimo de lucro (Gráfico 15). Las tareas que puede desarrollar un voluntario de cuidados paliativos en el domici-
lio, hospital o centro socio-sanitario son las siguientes:

-Acompañar al enfermo y su familia.

Establecer una comunicación activa con el enfermo y familia, ofreciéndoles es cucha y compresión.

Detectar necesidades y transmitirlas al resto del equipo.

- Sustituir al cuidador durante la acción voluntaria para facilitar el descanso físico
yemocional del mismo.

- Realizar pequeñas tareas o encargos de proximidad.

- Facilitar actividades lúdicas y de entretenimiento. 

Usted importa porque es usted

y, seguirá importando hasta el

último momento de su vida.

Nosotros, no sólo le ayudare-

mos a morir en paz, sino a vivir

hasta que muera.

Cicely Saunders

CONCLUSIONES

ATENCIÓN AL FINAL DE LA VIDA 
Usted nos importa a

nosotros y, entre todos,

intentaremos no fallarle

- Aliviar el sufrimiento es uno de los derechos básicos del ser humano

- Los diferentes sectores, especialidades y niveles asistenciales deben estar impli-
cados en la prestación de alivio al sufrimiento al final de la vida.

- La Atención Primaria es fundamental para garantizar la accesibilidad y eficiencia en la prestacion de Cuidados Paliativos, siendo tambien fundamental, la inter-
vención de los profesionales de Servicios Hospitalarios y de Centros Socio Sanitarios, donde existe una alta prevalencia de pacientes en situación de enferthe fors

- La formación específica en cuidados paliativos y una adecuada coordinación en tre los diferentes recursos asistenciales, incluidos los recurros especificos
dados paliativos, garantizan una atención de calidad al final de la vida. - La evaluación del proceso de atención, debe medir los resultados generales de
sistema, con la suma de todos sus componentes, siendo éste uno de los retos fu-

- El buen funcionamiento de un Programa de Cuidados Paliativos es mucho más los niveles implicados, y ahí está el desafío. 


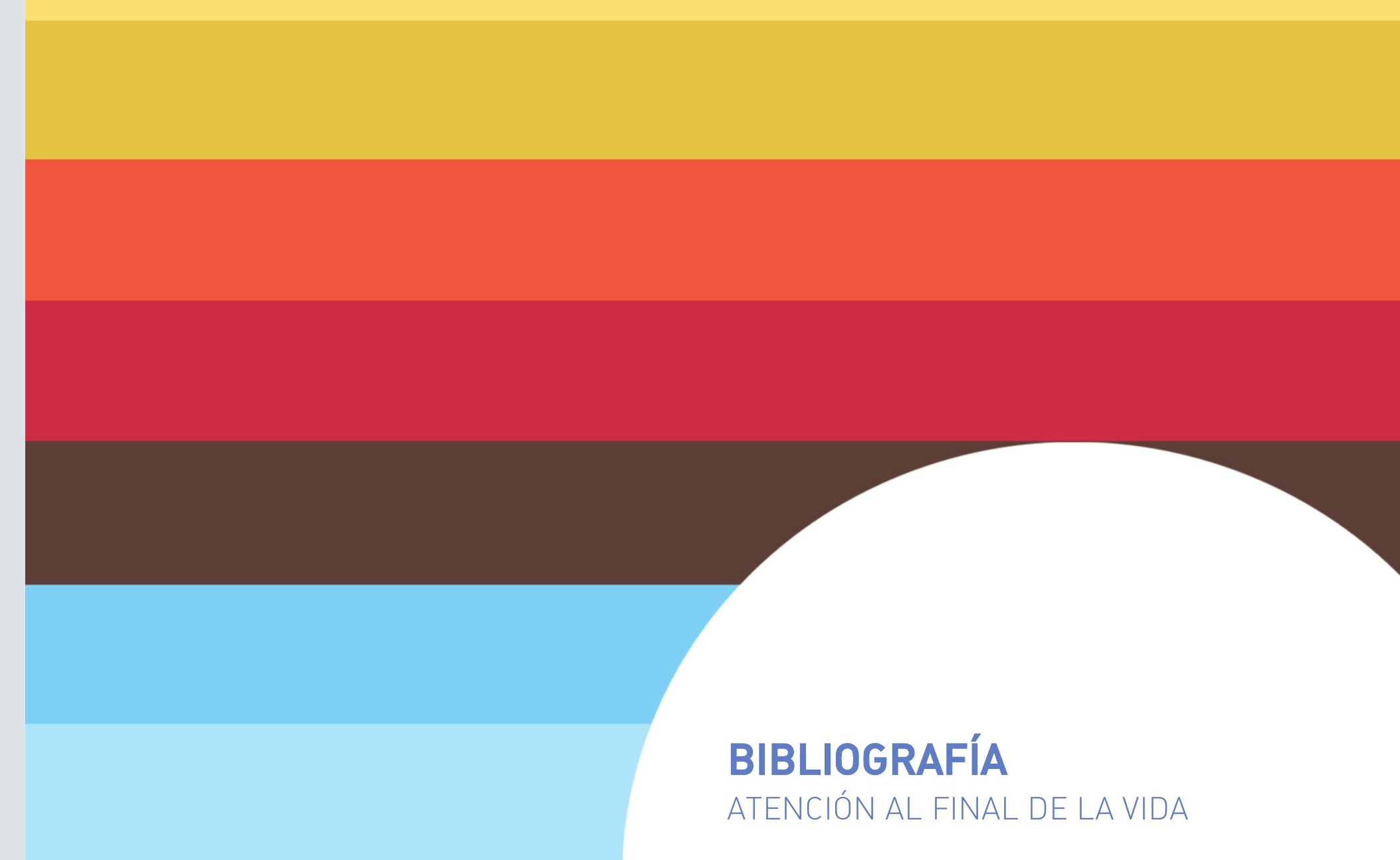


5.- Ley 16/2003, de 28 de mayo, de cohesión y calidad del Sistema Nacional de
Salud (BOE no 128, pag 20567-88, 29 de mayo de 2003)

6.- Plan Nacional de Cuidados Paliativos, 2001. Disponible en
httt://www.msc.es/organizacion/sns/plancalidadSNS/docs/PlanNacional.pdf

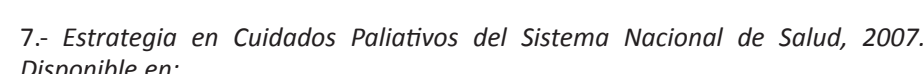

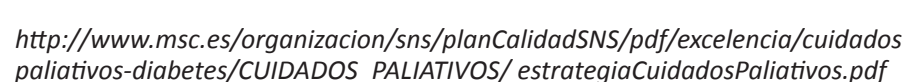




\section{DIRECTORIO DE RECURSOS ESPECÍFICOS}


DIRECTORIO Coordinación General del Programa de Cuidados Paliativos (PRCPEx)

Observatorio Regional de Cuidados Paliativos (ORCPEX)

Plan de Voluntariado del PRCPEX

ÁreAs DE SALUd

BADAJOZ

Hospital Perpétuo Socorro

CÁCERES

Hospital San Pedro de Alcántara

CORIA

Hospital Ciudad de Coria

DON BENITO - VILLANUEVA Hospital Don Benito-Villanueva

MÉRIDA Hospital de Mérida

NAVALMORAL DE LA MATA Hospital Campo Arañuelo

PLASENCIA Hospital Virgen del Puerto

LLERENA - ZAFRA Hospital de Zafr

EQUIPOS DE ATENCIÓN PSICOSOCIA

FUNDACIÓN OBRA SOCIAL LA CAIXA - FUNDESALUD

ATENCIÓN CONTINUADA Y DE URGENCA

SERVICIO DE ASESORAMIENTO TELEFÓNICO DE GUARDIA 


\section{DIRECTORIO}

PRCPEx, ORCPEx, ÁREAS DE SALUD EQUIPOS DE ATENCIÓN PSICOSOCIAL 
PROGRAMA REGIONAL DE CUIDADOS PALIATIVOS (PRCPEX)

COORDINACIÓN REGIONAL

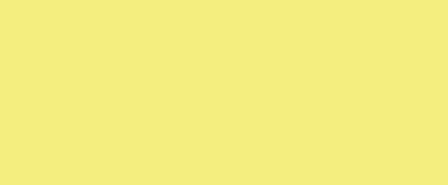

SERVICIO EXTREMEÑO DE SALUD (SES)
SERVICIOS CENTRALES

\begin{tabular}{l|l} 
Dirección & Avda de las Américas, 2. \\
20 &
\end{tabular}

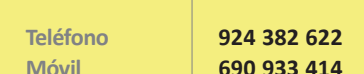

\begin{tabular}{l|l} 
Tel. Corporativo & 22622 \\
\hline
\end{tabular}

mail rafae

@gmail.com

rafael.motases.juntaextremadura.ne

\begin{tabular}{ll}
\hline Coordinador & D. Rafael Mota Vargas $\quad$ Médico. Servicio Extremeño de Salud
\end{tabular}

Auxiliar Administrativo
Servicio Extremeño de Salud
924382500 Extensión: 2850
OBSERVATORIO REGIONAL DE CUIDADOS PALLATIVOS (ORCPEX)

OBSERVATORIO REGIONAL

\begin{tabular}{l|l} 
Dirección & $\begin{array}{l}\text { Avda de las Américas, } 2 . \\
\text { 06800. Mérida }\end{array}$
\end{tabular}

SERVICIO EXTREMEÑN DE SALUd (SES)
SERVIICIOS CENTRALES

FUNDESALUD

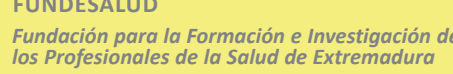

\begin{tabular}{l|l|l}
$\begin{array}{l}\text { Teléfono } \\
\text { Tel. Corporativo }\end{array}$ & 924382736 \\
222736
\end{tabular}

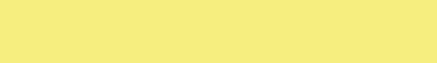

Email

Web

www.orceex.es

Director Técnico

Director Gerente

Técnico de Investigación
D. Rafael Mota Vargas

D. Felipe Sáez Tello

Da. María Solano Pallero
Médico. Servicio Extremeño de Salud

fundeEALUD

FUNDESALUD 
PLAN DE VOLUNTARIADO DEL PRCPEX

COORDINACIÓN DEL PLAN DE VOLUNTARIADO

SERVICIO EXTREMEÑO DE SALUD (SES)
SERVICIOS CENTRALES

PLATAFORMA DEL VOLUNTARIADO

ección Avda de las Américas, 2

\begin{tabular}{l|l} 
Teléfono & 924382500 \\
Extensión & 2831
\end{tabular}

Tel. Corporativo 22831

Email planvoluntariadocp@gmail.com

coordinador
Dạ. Yolanda Benítez Giles

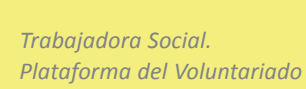

\begin{tabular}{|c|c|c|c|c|}
\hline NOMBRE ASOCIACIÓN & $\begin{array}{l}\text { COORDINADORA } \\
\text { VOLUNTARIADO }\end{array}$ & DIRECCIÓN & TELÉFONO & CORREO \\
\hline $\begin{array}{l}\text { PLATAFF VOLUNTARIADO } \\
\text { DE BADAOZZ }\end{array}$ & MARIBEL RUIZ & $\begin{array}{l}\text { AVDA. FEDERICO MAYOR } \\
\text { ZARAGOZA S SN BADAOZ }\end{array}$ & 924227016 & Plataformavoluntariado.ba@gmail.com \\
\hline $\begin{array}{l}\text { ASOCCIACIÓN CONTRA EL } \\
\text { CÁACER DE BADANOZ }\end{array}$ & LOLA Vázquez & $\begin{array}{l}\text { PLLZA DE PORTUGAL, 12. } 06001 \\
\text { BADANOZ. }\end{array}$ & 924236104 & | Doloresana.vazquez@aecc.es \\
\hline 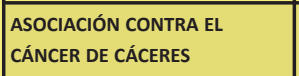 & INMACULADA DELGADO POLIO & $\begin{array}{l}\text { C/ DONANTES DE SANGRE, } 1 . \\
10002 \text { CACERES. }\end{array}$ & 927215323 & |Immaculada.delgado@aecc.es \\
\hline \begin{tabular}{|l|} 
ASOCIACIÓN ONCOLÓGICA DE \\
EXTREMADURA
\end{tabular} & RAQuel cano & $\begin{array}{l}\text { AVDA. GODOFREDO ORTEGAY } \\
\text { MUNOZZ, LOCAL 10. BADAOZZ }\end{array}$ & 924207713 & aoex@badajoz.org \\
\hline $\begin{array}{l}\text { PASTORAL DE LA DIÓCESIS DE } \\
\text { MERIDA-BADANOZ }\end{array}$ & JOSE M. ALVAREZ MAQUEDA & $\begin{array}{l}\text { CO OBISPO S. JUAN DE } \\
\text { RIBEAA, O6080 BADAOZ. }\end{array}$ & $\begin{array}{l}924255658 \\
64983652\end{array}$ & Joalma52@yahoo.es \\
\hline $\begin{array}{l}\text { PASTORAL DE LA SALUD CORIA- } \\
\text { CACERES }\end{array}$ & ANTONIO FLoRIANO & $\begin{array}{l}\text { PlaZZA DE SANTA MARRA, } 1 . \\
10001 \text { CACERES }\end{array}$ & 676340029 & afforico@telefonica.net \\
\hline $\begin{array}{l}\text { ASOCIACÓ́N PARKRINSON } \\
\text { ExTTEMADURA }\end{array}$ & ANA VELAZZQUEZ & 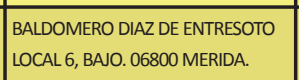 & 924303224 & Parkinsonextremadura@ hotmaili.com \\
\hline \begin{tabular}{|l} 
ASOCIACIÓN ExTREMEÑ̃̃ DE \\
LARINGECTOMIZADOS
\end{tabular} & AMala Franco & 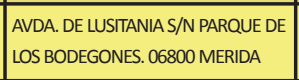 & $\begin{array}{l}924330067 \\
616809699\end{array}$ & Asociacionlaringectomizados@gmail.com \\
\hline $\begin{array}{l}\text { ASOC. FAM. ENFERMOS DE } \\
\text { ALZEHMER Y OTRAS... }\end{array}$ & Ma ÁNGELES GARRIDO & $\begin{array}{l}\text { C/ REYES HUERTAS S/N } \\
\text { O6880 MERRIDA }\end{array}$ & $\begin{array}{l}924009842 / 43 \\
\text { TS. } 635452469\end{array}$ & Afam8@hotmail.com \\
\hline \begin{tabular}{|l|} 
PASTORAL DE LA SALUD DE LA \\
DIOCESIS DE PLASENCIA
\end{tabular} & MARINA ALONSO & $\begin{array}{l}\text { PLAZAA DE LA CATEDRAL S/N } \\
10600 \text { PLASENCIA }\end{array}$ & 650304303 & $\begin{array}{l}\text { vicariopastorlediccesisplasencia.org } \\
\text { santosparedesm @vahoo.es }\end{array}$ \\
\hline 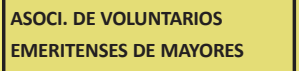 & LOLA DORADO & $\begin{array}{l}\text { C/REYES HUERTA S/N } \\
\text { O66800 MERIDA }\end{array}$ & $\begin{array}{l}924009842 / 43 \\
670970230\end{array}$ & |loladorado@ @otmail.com \\
\hline $\begin{array}{l}\text { ASOCCACIÓN ONCOOÓGICA } \\
\text { TIERAA DE BARROS }\end{array}$ & $\begin{array}{l}\text { LOURDES VIDAL } \\
\text { MAYTE DAAZ }\end{array}$ & $\begin{array}{l}\text { C/ LA HILRBA, } 9 . \\
\text { 19 ZIQUERRA }\end{array}$ & 924670723 & asoncologica@almendralejo.es \\
\hline $\begin{array}{l}\text { RED DE VOLUNTARIADO } \\
\text { DE GUAERENA }\end{array}$ & PETRA CARRASCO & $\begin{array}{l}\text { CENTRO SOCIOCUITURAL SAN } \\
\text { GiNES S/N0 } 06470 \text { GUARENAA }\end{array}$ & $\begin{array}{l}924351341 \\
625077212\end{array}$ & $\begin{array}{l}\text { Petri_carrasco@ @hotmail.com } \\
\text { Aromanno@@ hotmail.com }\end{array}$ \\
\hline FUNDACIÓN ICARO & NOELA SÁNCHEZ & $\begin{array}{l}\text { C/ RONDA DE MAESTRANZA, } \\
\text { 3-19 A. ZAFRA }\end{array}$ & 924552401 & antonio@fundacionicaro.org \\
\hline
\end{tabular}


EQUIPO DE CUIDADOS PALIATIVO
HOSPITAL PERPETUO SOCORRO

\begin{tabular}{l|c} 
Dirección & C/ Damián Téllez Lafuente, $5 / \mathrm{N}$. 06010. Badajoc
\end{tabular}

\begin{tabular}{l|l} 
Teléfono & $924215265 / 66$ \\
Fax & 924215267
\end{tabular}

Tel. Corporativo 45265 (Despacho general)

45266 (Auxiliar Administra

334655 (Rosario Peinado)
33466 (Miguel Angel Cuervo)

35617 (Miguel Angel Sáncher)
33242 (ME José Redondo)

ecuipal.badajoz@ses.juntaextremadura.net

\section{Coordinadora}

Médicos

Médico. Servicio Extremeño de Salud

Miguel Ángel Cuervo Pinna
D. Miguel Ángel Sánchez Correas

Servicio Extremeño de Salud
Servicio Extremeño de Salud

Dạ. Fátima Díaz Diez . Florencia Bonino Timmerman De. Yolanda Ruiz Castelllano

Servicio Extremeño de Salud
Servicio Extremeño de Salud

Enfermera

Da. Raquel Cabo Domínguez

Da. Elvira Menacho Perera

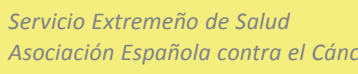

Psicóloga

Administrativa
Asociación Española contra el Cáncer

Servicio Extremeño de Salud
Dạ. Rosario Peinado Clemens

ÁREA DE SALU
EQUIPO DE CUIDADOS PALLATIVOS

\begin{tabular}{l|l} 
Dirección & $\begin{array}{l}\text { Avda. Pablo Naranjo s/n. } \\
\text { 10003. Cáceres. }\end{array}$
\end{tabular}

\begin{tabular}{l|l} 
& 927256217 \\
Teléfono & 92725217 \\
Fax & 927256217
\end{tabular}

\begin{tabular}{l|l} 
Tel. Corporativo & $51319 / 56323$ \\
Ma. Corporativo & $33905 / 3592$
\end{tabular}

Busca Hospital 31608

Email

ecuipal.caceres@ses.juntaextremadura.net
Coordinadora

Médicos

Enfermeras

Psicóloga
Dạ. Fabiola Saiz Cáceres

D. Ángel María Durán Martínez
Dạ. María Teresa Alonso Ruiz

Da. Rocío Valentín Tovar
D.. Teresa Galea Martín
Da. Victoria Romero Gabino

Dạ. Carmen López de Ayala García.
Médico. Servicio Extremeño de Salud

Servicio Extremeño de Salud
Servicio Extremeño de Salud

Servicio Extremeño de Salud
Servicio Extremeño de Salud
Servicio Extremeño de Salud 


\section{ÁreA DE SALUD}

CORIA

Dirección

Avenida de Cervantes, 75
10800. Coria.

EQUIPO DE CUIDADOS PALIATIV
HOSPITAL CIUDAD DE CORIA

\begin{tabular}{l|l} 
Teléfono & 927149200 (Centralita) \\
Fax & 927501898
\end{tabular}

\begin{tabular}{l|l} 
Tel. Corporativo $77369 / 77257$ \\
\hline
\end{tabular}

Email

ecuipal.coria@ses.juntaextremadura.net

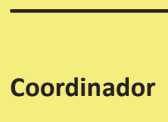

Médico

Enfermero

Psicólogo
D. Raúl Sánchez Posada

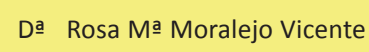

D. Antonio Fernández Romero

D. David Gámez García
Médico. Servicio Extremeño de Salud

Servicio Extremeño de Salud

Servicio Extremeño de salud

Asociación Oncológica Extremeña
ÁREA de Salud

DON BENITO - VILLANUEVA DE LA SERENA

EQUIPO DE CUIDADOS PALLAA
HOSPITAL DE DON BENTIO-
VILLANUEVA DE LA LERENA

Tel. Corporativo
Ma. Corporativo

Email

\begin{tabular}{ll}
\hline Coordinador & D. Raúl Pérez Asensio \\
\hline
\end{tabular}

Médico

Enfermeras

D. Marco Antonio López García

Servicio Extremeño de Salud

(1)

Da. Ana María Avuso Carmona
Da. María Eulalia Alonso Prado

Dạ. Petra González Cañamero

Servicio Extremeño de Salud
Servicio Extremeño de Salud

Asociación Oncológica Extremeño 
MÉRIDA

EQUIPO DE CUIDADOS PALLATIVOS
HOSPITAL DE MÉRIDA.

HOSPITAL DE MERRIDA.
$3^{3}$ PLANTA. BLOQUE POSTERIOR

\begin{tabular}{l|c} 
Dirección & $C /$ Poligono Nueva Ciudad, $s / n$. 06800. Mérida
\end{tabular}

\begin{tabular}{l|l|l} 
Teléf́no & 924381050 \\
Fax Compartido & 924382432
\end{tabular}

\begin{tabular}{l|l} 
Tel. Corporativo & 81050 (Despacho General) \\
81439 (Despacho Administrativo)
\end{tabular}

81439 (Despacho Ad

81437 (Consulta 2)
81338 (Consulta Psicol

81438 (Consulta P
$33631 / 33630$

\begin{tabular}{l|l} 
Email & ecuipal.merida@ses.juntaextremadura.net \\
\hline
\end{tabular}

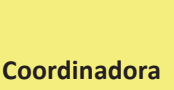

Dạ. Patricia Hernández García

Médico. Servicio Extremeño de Salud

Médico

Enfermeras

Psicóloga
Dạ. Mercedes Blanco Guerrero Servicio Extremeño de Salud

Da. Laura Blanco Toro
Dạ. Ma. Angeles Martin Fuente de la Rosas Servicio Extremeño de Salud
Servicio Extremeño de Salud

Dạ. Marta Gundin Martín
ÁREA DE SALUD

NAVALMORAL DE LA MATA

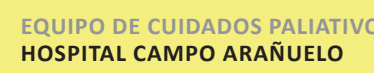

\begin{tabular}{l|l} 
Dirección & $\begin{array}{l}\text { C/ Del Hospital, } 5 / \mathrm{n} . \\
10300 \text { Navalmoral de la Mata }\end{array}$
\end{tabular}

\begin{tabular}{l|l} 
Teléf́nono & 927548900 \\
Fax (suministros) & 927588874
\end{tabular}

Tel. Corporativo $\quad 58873 / 58874$

$38873 / 586$
33818

ecuipal.navalmoral@ses.juntaextremadura.ne

Coordinadora $\quad$ Dạ. Beatrice Pop Csog Médico. Servicio Extremeño de Salud

Médico

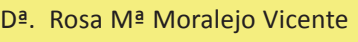

Servicio Extremeño de Salud
Tiempo compartido con Área de Coria

Enfermera

Da. Mercedes Gil Sierra

Servicio Extremeño de Salud

Psicóloga

Dạ. María Reyes Alcaide

Asociación Oncológica Extremeña 
PLASENEA DE SALU

EQUIPO DE CUIDADOS PALLATIVO
HOSPITAL VIRGEN DEL PUERTO

Dirección $\mid$ Paraje Valcorchero, $s / n$. 10600. Plasencia

\begin{tabular}{l|l|l}
\hline Teléf́ono & 927428300 \\
\hline Fax & 927421092
\end{tabular}

Tel. Corporativo 78514 (Dra. Paz Varillas López)

78167 (Dra. MMa Angeles Sánchez Pérez)

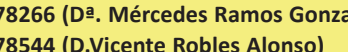

78168 (Consulta)
33413 / 33427

\begin{tabular}{l|l} 
mail & ecuipal.plasencia@ses.juntaextremadura.n \\
\hline
\end{tabular}

\section{Coordinadora}

Médico

Enfermeras

Psicólogo

\section{D’. Paz Varillas López}

Médico. Servicio Extremeño de Salud

Dạ. María Ángeles Sánchez Pérez

Servicio Extremeño de Salud

Da. Mercedes Ramos González

Servicio Extremeño de Salud
Servicio Extremeño de Salud

D. David Gámez García

$\begin{array}{ll}\text { AREA DE SALUD } & \\ \text { LLERENA-ZAFRA } & \text { Dirección } \\ \text { EQUiPO DE CUIDADDS PALLATIVOS } & \\ \text { HOSPITAL DE ZAFRA } & \text { Teléfono } \\ & \text { Fax } \\ & \text { Tel. Corporativo } \\ \text { Mo. Corporativo } \\ \text { Email } \\ \\ \end{array}$

/ C Carretera de Badajoz - Granada km 72.

924029200
924029251

40267 (Despacho/Consulta)

eccipal.llerena@ses.juntaextremadura.net

coordinador

D. Javier Flores Belmonte

Enfermero. Servicio Extremeño de Salud

Médico

Da. Yolanda Romero Cintas
Da. Milagros Cobián Prieto

Enfermera

Ḍ. María del Pilar Ruiz Márquez

Servicio Extremeño de Salud
Servicio Extremeño de Salud

Psicóloga

Dạ. Paloma Encinas Martínez
Servicio Extremeño de Salud

Asociación Oncológica Extremeña 
EQUIPO DE ATENCIÓN PSICOSOCIAL FUNDACIÓN OBRA SOCIAL LA CAIXA- FUNDESALUD

ÁREA DE SALUD DE CÁCERES

Centro Residencial "EI Cuartillo"
Avda/ de la Universidad $s / n$ 10003 Cáceres

CENTRO RESIDENCIAL “EL CUARTILLO
CENTRO CARE CACERES

\begin{tabular}{l|l}
$\begin{array}{l}\text { Móvil } \\
\text { Email }\end{array}$ & $\begin{array}{l}660051461 / 690925858 \\
\text { eaps@fundesalud.es }\end{array}$
\end{tabular}

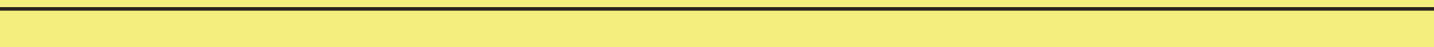

Coordinador

D. Manuel Carrasco Monroy

PSicólogo. FUNDESALUD

Psicólogos

D. Carlos García Martín

FUNDESALUD
FUNDESALUD

Trabajadora Social

Dạ. Begoña Majado Márquez

FUNDESALUD

ATENCIÓN

CONTINUADA Y DE URGENCIA

ASESORAMIENTO TELEFÓNICO DE GUARDIA 


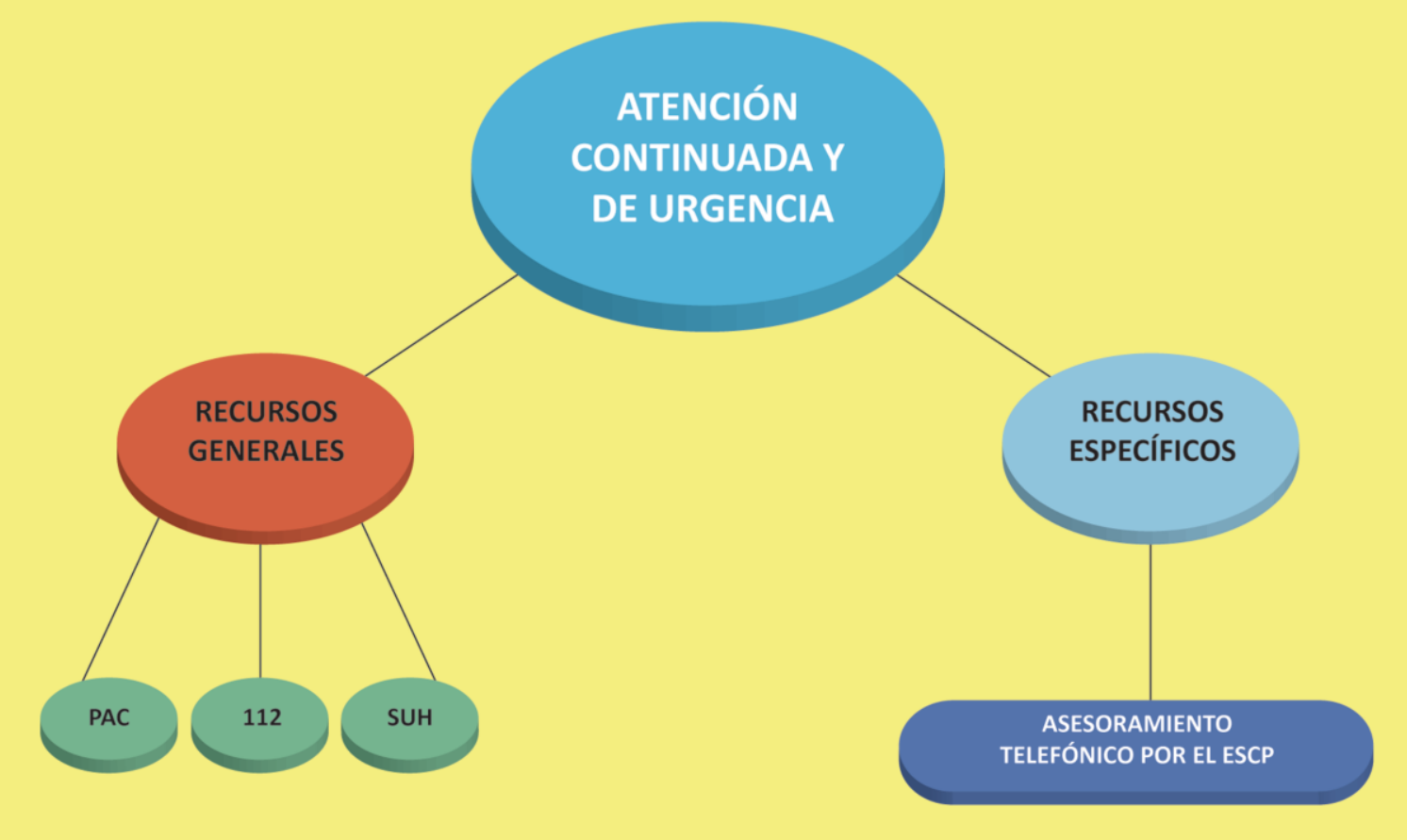

ATENCIÓN CONTINUADA DE URGENCI.

PROVINCIA DE BADAJOZ

Dís laborables: $15.00 \mathrm{pm} / 08.00 \mathrm{am}$
Fines de Semana y Festivos: $24 \mathrm{~h}$.

SERVICIO DE ASESORAMIENTO TELEFÓNICO

Horario

Tel. Corporativo 35811

Profesional
de Medicina

\begin{tabular}{l|l}
$\begin{array}{l}\text { Profesional } \\
\text { de Enfermeria }\end{array}$ & Tel. Corporativo 35813
\end{tabular}

PROVINCIA DE CÁCERES

Días laborables: $15.00 \mathrm{pm} / 08.00$ am
Fines de Semana y Festivos: $24 \mathrm{~h}$.

SERVICIO DE ASESORAMIENTO TELEFÉNICO
DE GUARDIA PARA PROFESIONALES

Horario

Tel. Corporativo 35812

\begin{tabular}{l|l}
$\begin{array}{l}\text { Profesional } \\
\text { de Enfermeria }\end{array}$ & Tel. Corporativo 35810
\end{tabular} 



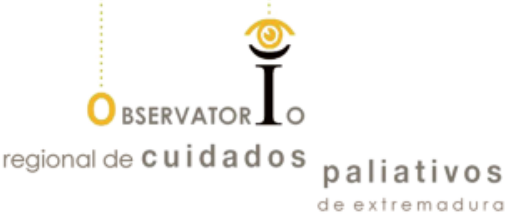

?ำ

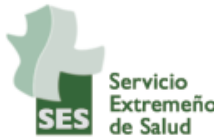

JUNTA DE EXTREMADURA

Consejería de Sanidad y Dependencia

- FundeSalud 